\title{
An Investigation of Omega Windfinding Accuracy
}

\author{
JAMES L. FRANKLIN \\ National Oceanic and Atmospheric Administration, Atlantic Oceanographic and Meteorological Laboratory, \\ Hurricane Research Division, Miami, FL 33149 \\ Paul R. Julian \\ National Center for Atmospheric Research, Boulder, $\mathrm{CO} 80303^{*}$
}

(Manuscript received 7 August 1984, in final form 26 December 1984)

\section{ABSTRACT}

\begin{abstract}
The major sources of error in Omega-derived wind estimates are examined and illustrated. Sample dropwindsondes and local Omega signals are used to illustrate the effects of several types of phase propagation anomalies. A stationary test sonde and synthetic Omega phases are used to determine the accuracy of three Omega phase-smoothing algorithms and their associated error estimates, and to determine the impact of base station motion for sondes released from aircraft.

Omega windfinding errors can be classified as either "internal" or "external" errors. Internal errors are associated with signal quality and transmitter-sonde geometry, while external errors are caused by anomalous phase propagation. Estimates of wind error (wind uncertainties) are provided by the equations of Omega windfinding. These uncertainties, however, estimate only the effects of internal errors. Precise assessment of errors caused by anomalous phase propagation requires the measurement of phase data by a stationary receiver. Such measurements show that errors from external sources range from about $1 \mathrm{~m} \mathrm{~s} \mathrm{~s}^{-1}$ for diurnal changes in ionospheric height to $20-30 \mathrm{~m} \mathrm{~s}^{-1}$ for sudden ionospheric disturbances. Methods for dealing with these problems in sonde postprocessing are described.

Data from a stationary test sonde show that the effect of aircraft maneuvers on real-time Omega wind estimates is substantial; during turns, errors in real-time wind estimates increase by over $50 \%$. The comparison of phase-smoothing algorithms shows that cubic-spline smoothing produces wind estimates $20-50 \%$ more accurate than those obtained with other methods. Hence, it is recommended that this smoothing algorithm be used in dropwindsonde postprocessing. It is estimated that such postprocessing will reduce errors by $60 \%$ during aircraft turns and by $30 \%$ at other times.
\end{abstract}

\section{Introduction}

Omega dropwindsondes (ODWs) are instruments used to obtain vertical profiles of pressure, temperature, humidity and wind from otherwise data-sparse regions. Over 5,000 ODW soundings are included in the First Global Atmospheric Research Program (GARP) Global Weather Experiment (GWE) data set (Julian, 1982). ODWs have been used in recent years over the Mediterranean Sea in GARP's 1982 Alpine Experiment (ALPEX), by the Atlantic Oceanographic and Meteorological Laboratory, Hurricane Research Division (HRD) to investigate the environmental flow of Hurricane Debby (1982), and by meteorologists to study the El Niño phenomenon in the eastern Pacific. Recently, researchers have been using ODWs in hopes of computing diagnostic quantities that require fairly precise measurements (El Niño, for example). ODWs have been used, or will be used in the near future, as input for important operational

- The National Center for Atmospheric Research is sponsored by the National Science Foundation. forecasting decisions regarding hurricanes and coastal winter storms, and they will continue to be a valuable tool until satellites can provide similar information. It is important, therefore, to understand the capabilities and limitations of this instrument. This study investigates and describes the major sources of error in ODW wind estimates.

To measure winds, ODWs use a network of eight very low frequency (VLF) transmitters, each of which broadcasts an approximately $1 \mathrm{~s}$ pulse of energy every $10 \mathrm{~s}$. Relative position of a sonde within an Omega lane can be calculated by measuring the signal phase of that station's transmission. As the sonde moves with the wind, Omega signals retransmitted from the sonde to a base station provide an estimate of the sonde's horizontal position. Successive position estimates are then used to estimate the horizontal wind. Sondes may be dropped from aircraft or from nearly stationary carrier balloons, or they may ascend from the ground like a rawindsonde. (In this paper, the term ODW is used to refer to both ascending and descending sondes.) In the case of balloon-born ascending sondes, it is feasible to record local VLF 
Omega at the launch site as well as the retransmitted signals. Such records are sometimes used for "differential" Omega windfinding (Acheson, 1974), but in this study we use local Omega to illustrate the effects of irregularities in Omega signal propagation. Although the emphasis here is on aircraft-launched ODWs, which present certain difficulties not found in systems using a stationary base station, most of the discussion is appropriate to Omega windfinding in general.

ODW windfinding errors can be described as either "external" or "internal." One of the assumptions of the basic windfinding equations is that the geometry defined by the eight Omega transmitters (i.e. the location and spacing of the Omega phase "lanes") remains essentially constant over the time interval necessary to determine sonde motion. Since it is impossible with sonde-transmitted signals to separate motion of the ODW from changes in the Omega lane geometry, violations of this assumption are considered to be external to the wind calculation. Olson et al. (1978) give a comprehensive inventory of these external error sources:

1) Phase modal interference,

2) diurnal changes in Omega propagation,

3) sudden ionospheric (solar-induced) disturbances,

4) long-path propagation and/or interference.

Given the assumption that the Omega lane geometry remains fixed, there are several other factors that influence wind accuracy. These "internal" factors, unlike the external factors listed above, influence accuracy in ways that are quantified automatically by the equations of Omega windfinding. These factors are:

1) Electronic system noise,

2) sferic noise,

3) sonde-transmitter geometry,

4) acceleration of the base station (aircraft turns),

5) choice of phase-smoothing algorithm.

Unlike conventional sonde tracking techniques that use radar or radiodirection finding, the errors in Omega windfinding are complex; but fortunately, they are largely quantifiable. The ODW data user should have an appreciation of these complexities so that the quantitative wind uncertainties (estimated wind errors) produced by ODW windfinding algorithms can be interpreted properly. It is our intent to supply the necessary considerations for that interpretation.

\section{Mathematical background}

\section{a. Basic equations}

The mathematics of Omega windfinding have been discussed in numerous contributions (Acheson, 1974;
Passi, 1974, 1977; Julian, 1982); hence only the basic outline necessary to the understanding of the discussion that follows is repeated here. The notation used follows Passi (1974).

Consider $k$ Omega stations $s_{j}, j=1, k$ at longitudes $a_{j}$ and latitudes $b_{j}$. If a dropwindsonde is at (longitude, latitude $)=(x, y)$, the central earth angle $f_{j}$ between station $j$ and the sonde is given by

$f_{j}=\cos ^{-1}\left[\cos \left(a_{j}-x\right) \cos (y) \cos \left(b_{j}\right)\right.$

$$
\left.+\sin (y) \sin \left(b_{j}\right)\right]
$$

and the distance between the station $j$ and the sonde is $r f_{j}$, where $r$ is the radius of the earth. The signal phase at the sonde at time $t, \phi_{s j}(t)$, is given by

$$
\phi_{s j}(t)=2 \pi r f_{j} / L+\omega\left(t-t_{1}\right),
$$

where $t_{1}$ is the time of zero phase at the transmitter, $L$ is the wavelength and $\omega$ is the radial frequency of the transmitted wave.

Omega signals are received by the sonde and retransmitted without modification to the launching aircraft. The signal phase at the aircraft, $\phi_{j}(t)$, will therefore be given by $\phi_{s j}(t)+c$, where $c$ is the change in phase that occurs between the sonde and the plane. However, $\phi_{j}$ is not the quantity measured; instead, a relative phase $p_{j}$ is measured, which is the difference of $\phi_{j}$ and the phase of a local oscillator. If we define $p_{i j}$ as $p_{i}-p_{j}$, the phase difference between stations $i$ and $j$, it can be shown easily that this difference of relative phases is equal to the difference of Omega signal phases $\phi_{i}-\phi_{j}$. It then follows that

where

$$
p_{i j}=(2 \pi r / L) f_{i j} \text {, }
$$

$$
f_{i j}=f_{i}-f_{j}, \quad i \neq j=1, k .
$$

The $p_{i j}$ are referred to as the hyperbolic phases, for reasons that shall be clear momentarily. Rearranging (3) gives

$$
\left(r f_{i}-r f_{j}\right)=(L / 2 \pi) p_{i j}, \quad i \neq j=1, k
$$

Measurements allow the calculation of the righthand side of (4). Now the set of points that satisfies $\left(r f_{i}-r f_{j}\right)=$ constant describes a hyperboloid. A second hyperbolic phase measurement, $p_{i m}, m \neq j$, defines another hyperboloid, whose intersection with the first is a (nearly) vertical line. In this manner, Omega signals from three stations define the $(x, y)$ position of the sonde.

Only $k-1$ equations of (3) are independent. With no loss, therefore, we may set $j=k$ :

$$
p_{i k}(t)=(2 \pi r / L) f_{i k}, \quad i=1, k-1 .
$$

Differentiation with respect to $t$ gives

$$
\dot{p}_{i k}=\frac{\partial p_{i k}}{\partial t}=\frac{2 \pi r}{L}\left\{\frac{\partial f_{i k}}{\partial x} \frac{\partial x}{\partial t}+\frac{\partial f_{i k}}{\partial y} \frac{\partial y}{\partial t}\right\}
$$


and since $U=r \cos (y) \partial x / \partial t$ and $V=r \partial y / \partial t$, substitution gives

$$
\begin{aligned}
\dot{p}_{i k}=[2 \pi / L \cos (y)] & \frac{\partial f_{i k}}{\partial x} U \\
& +(2 \pi / L) \frac{\partial f_{i k}}{\partial y} V, \quad i=1, k-1
\end{aligned}
$$

or in vector notation,

$$
\dot{\mathbf{p}}=\mathbf{F}\left[\begin{array}{l}
U \\
V
\end{array}\right]
$$

Omega phase data typically contain noise that makes the evaluation of $\dot{p}$ difficult. For this reason, $\dot{\mathbf{p}}$ is generally represented as $\dot{\mathbf{p}}+\mathbf{s}$, where $\dot{\mathbf{p}}$ is the phase-rate estimator matrix and $\mathbf{s}$ is the matrix of phase-rate difference errors. Equation 8 then becomes

$$
\dot{\mathbf{p}}+\mathbf{s}=\mathbf{F}\left[\begin{array}{l}
U \\
V
\end{array}\right] .
$$

For $k$ Omega stations, (9) represents $k-1$ independent equations in two unknowns. Thus, for $k>3$, the system is overdetermined and the traditional solution (Julian, 1982) is

$$
\mathbf{U}=\left(\mathbf{F}^{T} \mathbf{E}^{-1} \mathbf{F}\right)^{-1} \mathbf{F}^{T} \mathbf{E}^{-1} \dot{\mathbf{p}}
$$

where $\mathbf{E}$ is the covariance matrix of $\mathbf{s}$, and $\mathbf{U}=[U$, $V]^{T}$, and $\left(\mathbf{F}^{T} \mathbf{E}^{-1} \mathbf{F}\right)^{-1}$ represents the covariance matrix of $U$ (Passi, 1977). The estimated wind error due to internal sources of error, or quantitative wind uncertainty (WU), is defined by (Var $U+\operatorname{Var} V)^{0.5}$ and is thus given by the square root of the trace of $\left(F^{T} E^{-1} \mathbf{F}\right)^{-1}$ (Passi, 1973).

Since $\mathbf{s}$ is made up of phase rates, the elements of E should come from estimates of observed phase-rate errors. In general, however, no closed form expression for phase-rate errors exists, so $E$ must be obtained some other way. Passi argues that the covariance matrix E computed from phase-rate errors should be proportional to one computed from phase errors alone. This proportionality constant would fall out of (10) and not influence $U$, although it would affect WU. The covariance matrix $\mathbf{E}$ suggested by Passi has its elements given by

$$
e_{i j}= \begin{cases}s_{i}^{2}+s_{k}^{2}, & \text { for } i=j \\ s_{k}^{2}, & \text { for } i \neq j,\end{cases}
$$

where the phase variance $s^{2}$ is given by

$$
\begin{aligned}
s^{2}=\sum_{i=1}^{n} K\left[p\left(t_{i}\right)-\hat{p}\left(t_{i}\right)\right]^{2} / \\
\\
\quad(n-\# \text { of parameters estimated). }
\end{aligned}
$$

$K$ is the proportionality constant referred to above and $n$ is the total number of phase observations. In the remainder of Section 2 we describe the methods used to estimate the phase rates $\hat{\dot{p}}$ and the constant $K$.

\section{b. Phase-smoothing algorithms}

Given the assumption that the hyperbolic phase lane pattern through which the sonde is moving remains steady during sonde motion, several sources of wind estimate error remain. Two of these sources are electronic system noise and sferic noise. The former is associated with the system's transmitters, receivers and antennas, as well as any nearby electronic equipment; the latter with radio frequency effects of lightning discharges that cause $\sim 1 \mathrm{~Hz}$ interference with the Omega signals. In ODW equipment, the latter noise source is effectively filtered electronically. The internal system noise, however, is important, and the ability of the ODW system to determine accurate phase rates depends upon the signal-to-noise ratios for the sonde-retransmitted Omega. The noise filtering problem and the necessity of phase smoothing have been described by Govind (1975). Since decorrelation times for typical ODW noise are tens of seconds or less, phase measurements taken $10 \mathrm{~s}$ apart must be smoothed by some technique to eliminate the higher frequency noise. Considerations of the noise characteristics of ODW equipment by previous investigators (Acheson, 1974; Govind, 1975) suggest that averaging or smoothing times of 2-4 min are necessary to reduce the error of ODW wind vectors to a few meters per second. A variety of smoothing methods have been used in ODW applications; three of the most common are quadratic smoothing, low-pass digital filtering, and cubic-spline smoothing.

The simplest of the three methods is quadratic smoothing: a second order, least-squares polynomial approximation to the phase data. Because of its simplicity, this routine is used to compute real-time winds for aircraft-launched ODWs. In addition, this routine has been used in most of HRD's postprocessing of ODW data before 1984 .

To estimate phases at a time $t_{0}$, a quadratic is fit to $3 \mathrm{~min}$ of phase data (19 phase measurements) surrounding $t_{0}$ by the method of least-squares. A fit is done for each of the Omega stations used in the computation. Once the fitting polynomials are determined, they are analytically differentiated to obtain phase rates at $t_{0}$. Phase variances are evaluated using residuals from the polynomial fit in (12) with $n=19$. This procedure is then repeated for time $t_{0}+10$ with the next phase measurement at the center of a new 3 min window.

There are several limitations of this quadratic model. As a practical matter, since each wind evaluation occurs at the midpoint of a 3 min phase window and the sonde falls at $25-35 \mathrm{mb} \mathrm{min}^{-1}$, no winds can be computed within about $40 \mathrm{mb}$ of launch and 50 
$\mathrm{mb}$ of sonde splashdown. No direct estimate of surface winds is therefore available. There are theoretical limitations as well. The quadratic approximation implies a constant component of acceleration toward each Omega station over a $3 \mathrm{~min}$ interval. For a sonde moving with the wind, this is a reasonable approximation; however, the measurement of signal phase takes place on a moving aircraft. When the aircraft turns, this restriction of constant acceleration implied by the quadratic is not satisfied. Another problem with least-squares quadratic smoothing is the sensitivity of the fitted curve's slope to points near the ends of the fit. Irregularities in phase data due to noise or aircraft manuevers can therefore have a large impact on computed winds as they enter and exit the 3 min smoothing window.

A second smoothing method employs low-pass filtering of Omega data and is described by Julian (1982). This algorithm takes the entire phase sequence for each station and transforms it into frequency space using a fast Fourier transform. The transform is then multiplied by a low-pass filter function, a complex exponential $\exp \left(-i \omega r_{j}\right)$ to correct for the transmission time difference between stations $\left(r_{j}\right.$ is the relative time lag of station $j$ 's transmission within the $10 \mathrm{~s}$ transmission cycle), and by $i \omega$ to produce phase rates from phases. After experimentation with radar-tracked ODWs, Julian settled on a filter with a 4 min effective length.

One would expect this procedure to be less susceptible to unedited random noise than a quadratic because much of the noise is explicitly filtered and there are no fixed smoothing windows. Low-pass filtering has been used in HRD processing primarily for sondes with relatively poor Omega; much less erratic wind profiles resulted from this algorithm than from quadratic fits for these sondes. Filtered phases are not computed in the $60-70 \mathrm{mb}$ layers at the ends of the sounding; these "blacked-out" layers are slightly larger than those resulting from quadratic smoothing. The low-pass filter has been, nonetheless, an excellent alternative to the quadratic smoother for poorer quality signals.

The third algorithm examined in this study involves cubic-spline smoothing of phase data (Passi, 1974). This method was used to process ALPEX ODW data at the National Center for Atmospheric Research (NCAR) and has recently been implemented at HRD. The routine first divides the entire phase sequence into segments of about $3 \mathrm{~min}$. Cubic polynomials are then fit to each segment, with the restriction that adjacent cubics give the same phases, phase rates and radial accelerations at the join points (nodes).

Cubic-spline smoothing uses more information than that contained in any $3 \mathrm{~min}$ interval alone; thus continuity of the wind field is allowed to influence the solution. One would expect aircraft maneuvers to be better handled by the cubic polynomials than the quadratic, and the continuity restrictions should eliminate the quadratic's window effects. However, the effect of continuity restrictions on the spline's resolution is not yet established.

In Section 4 the performance of each of the three phase-smoothing algorithms described above is evaluated.

\section{c. Calibration of wind uncertainties}

In Section $2 \mathrm{a}$, the process of estimating the uncertainty in the calculated wind vectors from results of the phase-smoothing procedure was introduced. Passi (1974) argues that except in the case of quadratic smoothing, the constant relating the phase and phaserate error matrices must be determined empirically. This procedure makes use of simultaneous measurements of the horizontal sonde displacement by means of precision radar tracking and the ODW algorithm.

For the algorithms described in the previous section, FPS-16 radar tracking during 1978 at Patrick Air Force Base, Florida, was used to determine the scaling constant and thus calibrate the quantitative uncertainties of the ODW. winds. In particular, the trace of the $\left(F^{T} E^{-1} F\right)^{-1}$ matrix in (10) is varied by multiplication of the elements of $E$ by the scalar constant, $K$, until the vector variance, radar-ODW wind, is approximately twice the trace:

$$
\frac{\left|\mathbf{V}_{R}-\mathbf{V}_{0}\right|^{2}}{2} \approx K\left[\operatorname{Tr}\left(\boldsymbol{F}^{T} \mathbf{E}^{-1} \mathbf{F}\right)^{-1}\right]
$$

This procedure assumes that half of the error variance in the wind measurements is ascribed to the radar and half to the Omega-determined winds. This distribution of error is based on a subjective assessment of the noise in the radar winds, since the actual radar capabilities are not well known. Omega wind errors are probably underestimated, although the results of Section 4 indicate a revised distribution is unnecessary.

Typical results of these calibrations are shown in Table 1. Two drops from an April 1980 test program are included: one with strong winds and little directional shear; and one with weaker and more variable winds. These drops should be independent tests of the calibration, since the scaling constant used was determined during the 1978 tests. The transmittersonde geometry is excellent (Fig. 1) and overall Omega signal quality good. (Good geometry is obtained by maximizing the angular separation of the Omega stations while avoiding the use of antipodal stations; for example, the three-station combination of Hawaii, Norway, and Argentina is excellent for south Florida.) The table includes a comparison of the wind vectors, the vector difference, the ODW wind uncertainty (WU) and the vector difference divided by $\sqrt{2}$ for comparison with WU. The ODW winds were estimated by the low-pass filter algorithm, and the radar winds were smoothed over approxi- 
TABLE 1. Sample radar-ODW calibration, Patrick AFB, Florida. Units are meters per second for speed, degrees for direction.

\begin{tabular}{|c|c|c|c|c|c|}
\hline $\begin{array}{c}P \\
(\mathrm{mb})\end{array}$ & ODW & $\begin{array}{l}\text { Radar } \\
\text { wind }\end{array}$ & $\begin{array}{l}\text { Vector diff. } \\
\text { (ODW-Radar) }\end{array}$ & $\frac{\mathrm{WU}}{K\left[\operatorname{Tr}\left(\boldsymbol{F}^{T} E^{-1} \mathbf{F}\right)^{-1}\right]^{1 / 2}}$ & $\begin{array}{c}\text { WU } \\
\text { (Vector diff. } / \sqrt{2} \text { ) }\end{array}$ \\
\hline
\end{tabular}

$\begin{array}{ll}500 & 233 / 42.4 \\ 600 & 231 / 34.6 \\ 700 & 238 / 32.9 \\ 800 & 270 / 20.5 \\ 850 & 288 / 17.3 \\ 900 & 289 / 11.0 \\ 950 & 308 / 8.5 \\ \text { WU: } & \end{array}$

$\begin{array}{ll}231 / 40.2 & 2.7 \\ 232 / 36.6 & 2.0 \\ 240 / 29.5 & 3.6 \\ 270 / 23.5 & 3.0 \\ 288 / 16.2 & 1.1 \\ 297 / 11.1 & 1.5 \\ 314 / 7.6 & 1.2\end{array}$

0.6
1.2
1.8
0.7
0.6
0.6
0.6
0.87

1.9
1.4
2.5
2.1
0.8
1.1
$\frac{0.8}{1.51}$

18 April 1980, Sonde Number 14014

$\begin{array}{lll}500 & 252 / 25.0 & 254 / 21.6 \\ 600 & 253 / 13.2 & 252 / 14.7 \\ 700 & 306 / 4.0 & 292 / 3.9 \\ 800 & 087 / 4.6 & 079 / 4.0 \\ 850 & 055 / 4.4 & 076 / 3.2 \\ 900 & 151 / 2.6 & 151 / 2.2 \\ 950 & 108 / 6.4 & 111 / 5.2 \\ \text { WU: } & & \end{array}$

$\begin{array}{lll}3.4 & 1.1 & 2.4 \\ 1.5 & 1.3 & 1.1 \\ 0.9 & 1.5 & 0.6 \\ 0.9 & 1.4 & 0.6 \\ 1.8 & 0.3 & 1.3 \\ 0.4 & 0.8 & 0.3 \\ 1.2 & \underline{0.8} & \frac{0.8}{1.01} \\ & \underline{1.03} & \end{array}$

mately 3 min using a digital filter with binomial weights to produce comparable vertical resolution in the two data sets.

Table 1 shows that the scaling is satisfactory overall, and that with good signal quality and transmittersonde geometry the ODW system is capable of measuring winds with uncertainties on the order of 1-2 $\mathrm{m} \mathrm{s}^{-1}$. However, the average vector differences for the 15 April case are 50\% larger than those for the

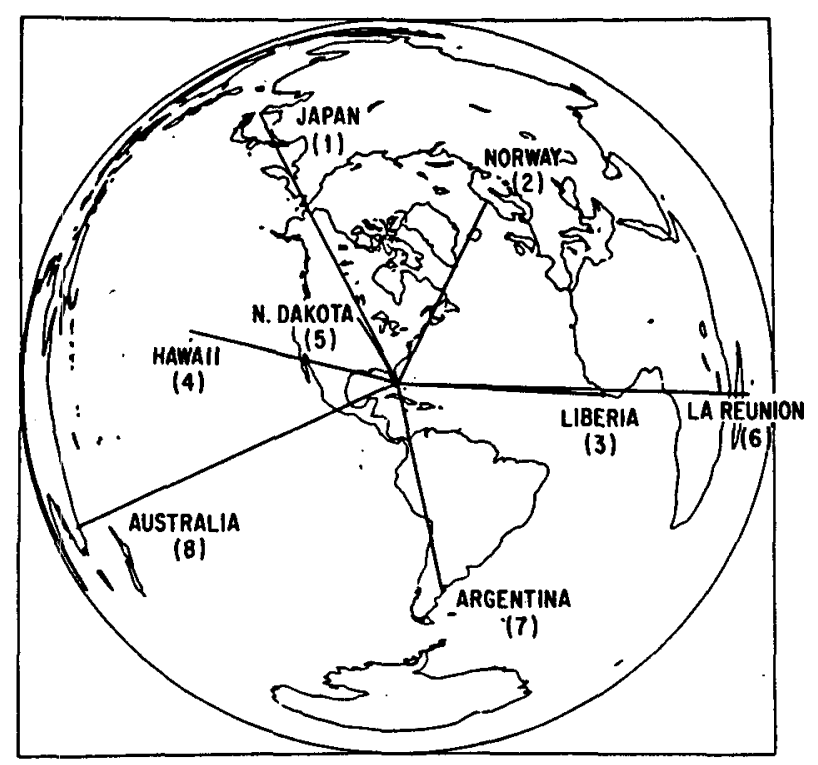

FIG. 1. An equal-areal projection centered on the location of the Patrick AFB test ODW on 15 April 1980. Great circle arcs to each of the Omega transmitters are shown to illustrate the sondetransmitter geometry.
18 April drop, but the WU values are $20 \%$ larger for the latter. Furthermore, the largest (smallest) WU do not always correspond with the largest (smallest) vector differences. These discrepancies do not necessarily indicate that the uncertainties are of little value; in theory, they do account for the geometry of the hyperbolic sonde tracking as well as the signal quality. The utility of the wind uncertainties is investigated further in Section 4e.

\section{Effect of external factors}

As noted in Section 1, the accuracy of a wind vector calculated from hyperbolic Omega navigation depends upon the steadiness of the Omega lane geometry. In other words, the $f_{j}$ are assumed to be proportional to the sonde-station separation distance, and this proportionality is assumed to remain constant in space and time. External errors caused by time variations in the lane geometry are discussed below. There is one type of external error caused by a spatial variation, rather than a time variation in geometry: within about $800 \mathrm{~km}$ of an Omega transmitter, the VLF signal propagation occurs with a mixture of the lowest and higher harmonics. Thus the Omega lane widths are not constant and given uniquely by the transmitted frequency. Fortunately, this "phase modal interference" is serious only relatively near the transmitter and the problem is simply overcome by omitting any nearby station from the calculations.

\section{a. Diurnal variation}

VLF signal propagation depends principally upon the configuration of the ionospheric layer that reflects the Omega signals (Watt, 1967). The diurnal variation 
in solar radiation produces a change in the height of the ionosphere, which alters the phase lane geometry of the Omega signals. By continuously recording local Omega with a very stable stationary receiver, the diurnal variation in Omega phase may be determined empirically. Figures $2 \mathrm{a}-\mathrm{c}$ are samples of $13.6 \mathrm{kHz}$ Omega signals received by NCAR over a 3-day interval during the GWE. A rubidium standard reference oscillator was used for long-term stability. Phase data at hourly intervals during the 3-day period have been plotted in centicycles $[100$ centicycles (cec) $=2 \pi$ radians] as a function of Local Standard Time (LST). Successive days have been plotted on the same scale with the days as marked. The traces reflect three different path configurations: Fig. 2c represents a relatively short path length with transmitter and receiver at approximately the same longitude, while Figs. $2 a$ and $b$ are taken from longer paths with the transmitter to the west and east of the receiver respectively. At night, ionospheric conditions are relatively stable and the hourly phases are fairly constant. When the terminator impinges on the transmission path at sunrise, the phase begins to change rapidly. At local noon of the transmission path midpoint, the extreme value of phase is reached. Maximum phase rates during sunrise are $\sim 20 \mathrm{cec} \mathrm{h}^{-1}$ or about $6 \times 10^{-3} \mathrm{cec} \mathrm{s}^{-1}$. An estimate of the effect of such spurious phase rates can be obtained by multiplying the width of the Omega lanes by the magnitude of the phase rates:

$$
\frac{22000 \mathrm{~m}}{100 \mathrm{cec}} \times \frac{6 \times 10^{-3} \mathrm{cec}}{1 \mathrm{~s}} \sim 1 \mathrm{~m} \mathrm{~s}^{-1} .
$$

Although this error is not as large as errors from other sources, it can easily be corrected by modeling the diurnal phase cycle. A detailed model accounting for path geometry, solar inclination and other relevant variables is possible, but we can use a much simpler scheme that still retains the characteristic daylight shape of the diurnal phase profile. Our simple model is a hyperbola determined by two parameters: the amplitude (the difference between nighttime and noon values) is set proportional to the Omega station-tosonde great circle path distance; the base width, or time between terminator impingement and departure, is set proportional to the longitudinal (time) difference between the sun and the path midpoint. Sample curves, such as in Fig. 2, are used to estimate these parametric constants. This model produces phase rates that satisfactorily reproduce those observed.

\section{b. Sudden ionospheric disturbances}

The steadiness of the VLF waveguide mode propagation over time scales of seconds to hours generally is sufficient so that it is not a consideration in the accuracy of ODW winds. However, a sudden ionospheric disturbance (SID) resulting from solar flare or geomagnetic activity may disturb the ionospheric waveguide on time scales that do affect the calculated winds. Figure 3 shows local Omega signals taken from the chart record on Canton Island on 5 February 1979 for three of the $13.6 \mathrm{kHz}$ Omega transmitters. At 2120 GMT (0920 LST) an SID was observed and Omega propagation for the stations being recorded at Canton was severely affected. The three stations shown exhibited typical phase disturbances with a very rapid rise time, the largest being $\sim 30-40 \mathrm{cec}$ in $5 \mathrm{~min}$, followed by a slower recovery. The total disturbance time was $\sim 1 \mathrm{~h}$. The spurious phase rates $\left(\sim 0.1 \mathrm{cec} \mathrm{s}^{-1}\right)$ produced by this SID might be expected to produce wind errors of about

$$
\frac{22000 \mathrm{~m}}{100 \mathrm{cec}} \times \frac{0.1}{\mathrm{l}} \frac{\mathrm{cec}}{\mathrm{s}}=22 \mathrm{~m} \mathrm{~s}^{-1} \text {. }
$$

Figure 4 presents a sequence of three wind profiles taken during this SID. The left panel is from a sonde dropped prior to the SID and represents an undisturbed sounding. The effect of the SID can be seen in the center and right profiles; the errors induced by the SID are about $25 \mathrm{~m} \mathrm{~s}^{-1}$. Further, it may be noted that the total portion of the center profile seriously affected by the SID was $\sim 150 \mathrm{mb}$ or, with a fall rate of $25 \mathrm{mb} \mathrm{min}^{-1}, \sim 6 \mathrm{~min}$. From Figs. 3 and 4 , it is obvious that the large spurious phase rates from 2120 to $2130 \mathrm{GMT}$ are producing the wind speed anomaly, and that the recovery portion of the disturbance, with spurious phase rates an order of magnitude less, has much less disastrous consequences for the wind profile.

There is no method for detecting spurious phase rates in the windfinding equations; however, there are at least two possible remedies to the SID problem. First, if local (not sonde-retransmitted) Omega is available (as, for example, in Fig. 3), the SID-induced phase rates can be measured and removed from the sonde-transmitted signals. This laborious process is dependent upon having locally received Omega for all stations used in the windfinding process and the processing capability of handling that data. In aircraft ODW systems, locally recorded Omega is not available. Some progress has been made, however, in modeling the SID-induced phase rates. In these models, the phase-rate signature (Fig. 3) is predicted as a function of solar flare time, intensity and Omega path length, weighted by solar zenith angle. These models have not been tested with ODWs, but could conceivably be used in future ODW postprocessing.

\section{c. Long-path interference}

One important characteristic of VLF propagation is that signal strengths for daytime eastward propagation are greater than for westward propagation. The region in which interference of the signals may be expected is thus not antipodal to an Omega station, but shifted eastward. Estimates of this displacement of the interference region, prior to ODW experience in FGGE, were $\sim 20-40^{\circ}$. Station selection 

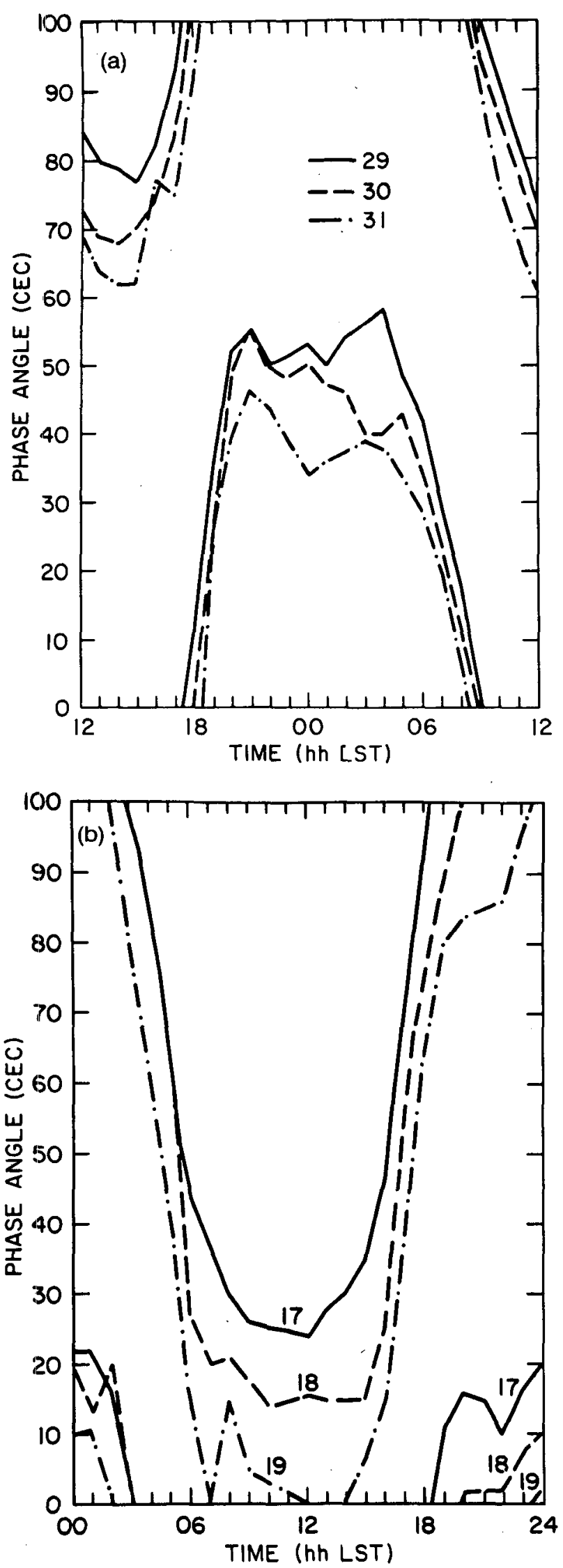

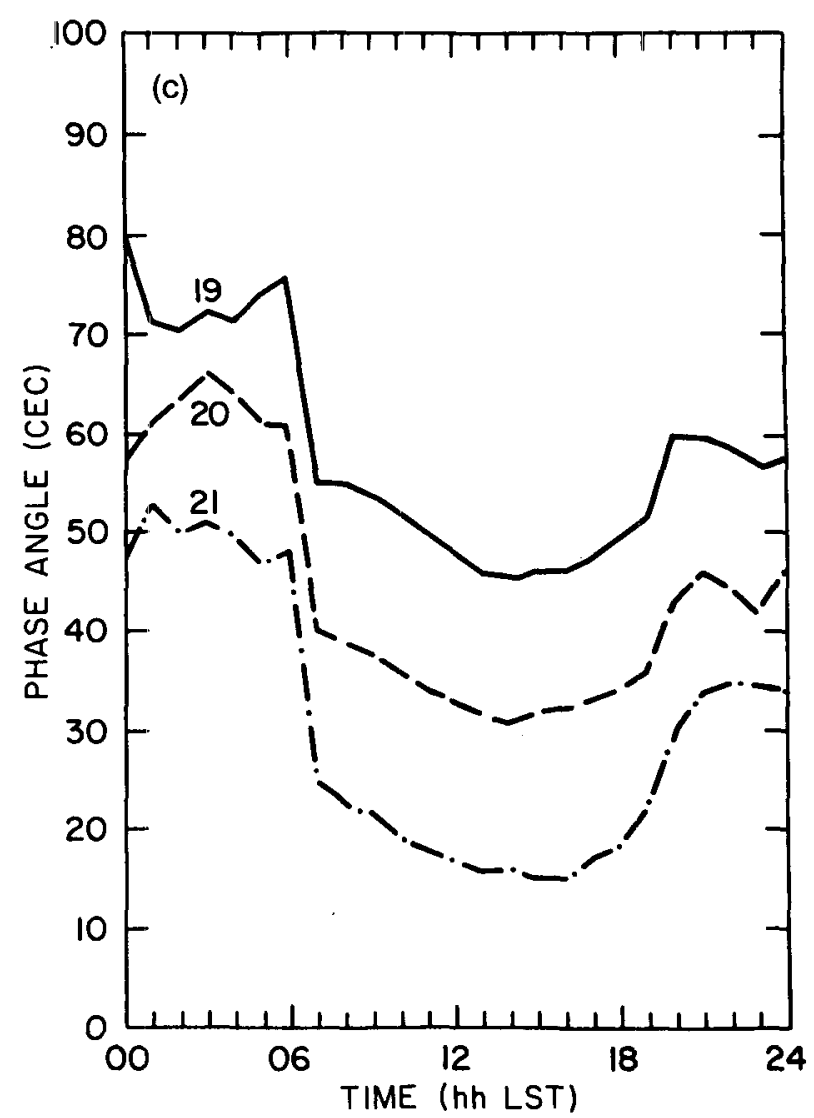

Fig. 2. Relative phase, in centicycles (cec), of the (a) Japan Omega signal recorded for three successive days (29-31 January $1979)$ at Canton Island $\left(3^{\circ} \mathrm{S}, 172^{\circ} \mathrm{W}\right)$, (b) LaReunion signal for 17-19 February 1979 , recorded at Ascension Island $\left(8^{\circ} \mathrm{S}, 14^{\circ} \mathrm{W}\right)$ and (c) Liberia signal for 19-21 January 1979, also recorded at Ascension Island. Data each hour were extracted from the original chart record made by an ultrastable receiver (rubidium standard oscillator). The abscissa is approximate Local Standard Time (LST) of the receiver. 


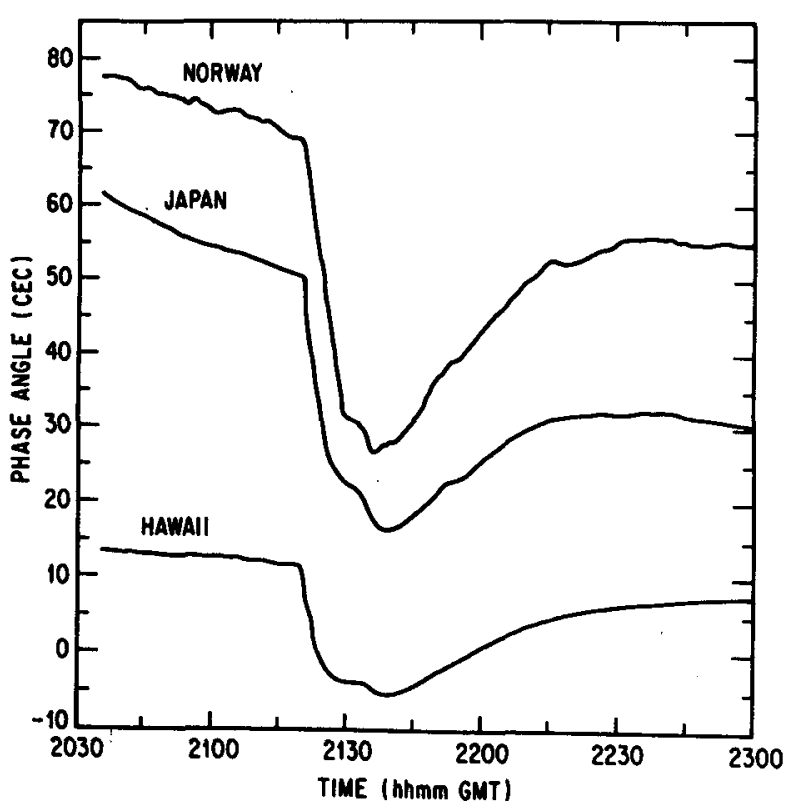

FiG. 3. Relative phase (cec) of the Norway, Japan and Hawaii transmitters received at Canton Island on 5 February 1979. guidelines thus specified that signals coming from the east should not be used if great circle distances were greater than about $140^{\circ}$. Examination of GWE ODW wind profiles in tropical regions revealed that the situation was actually more complicated and that, on occasion, long-path (eastward) propagation could occur to distances $>240^{\circ}$.

One way to detect this anomalous propagation is by estimating winds with and without a suspect Omega signal. Tables 2 and 3 illustrate the disastrous effect of using an Omega signal propagating along the long-path geometry. Two sample drops in the Arabian Sea from the summer MONEX period are shown. In these drops Hawaii's signal is propagating long-path. Note that Japan and Hawaii's Omega transmitters lie on nearly the same azimuth (heading) from the sondes. Table 3 shows the $850 \mathrm{mb}$ wind vector and quantitative wind uncertainty, WU, estimated using various combinations of Omega signals. (Recall that the WU assume normal, or short-path signal propagation.) The $850 \mathrm{mb}$ wind vectors computed using Hawaii's signal show a' great deal of variability relative to $\mathrm{WU}$, while those not using

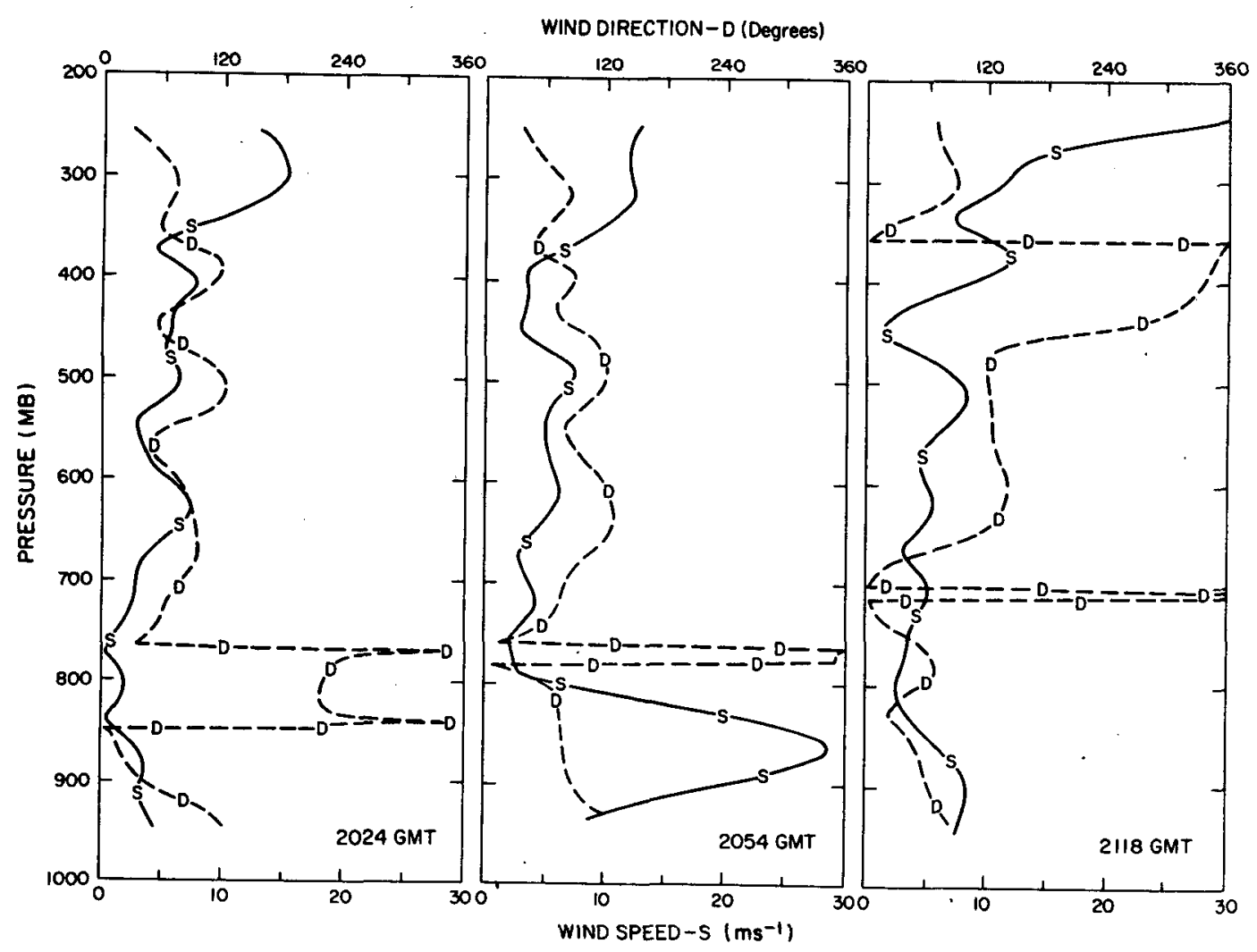

Fig. 4. Omega wind profiles from three successive drops in the central Pacific on 5 February 1979. The ordinate is linear pressure and the abscissa is wind speed (bottom) and wind direction (top); the speed and direction profiles are marked. The left panel was from a drop launched at 2024 GMT, the middle from one at 2054 GMT and the right from one at $2118 \mathrm{GMT}$. These launch times appear in each panel; from the initial height of $250 \mathrm{mb}$, these sondes took about $35 \mathrm{~min}$ to reach the surface. A sudden ionospheric disturbance occurred at 2120 GMT (Fig. 2). 
TABLE 2. Omega station-sonde geometry for drops A and B (see Table 3). Units are degrees from north for heading and degrees of arc for distance.

\begin{tabular}{|c|c|c|c|c|c|c|c|c|c|c|c|c|c|c|c|c|}
\hline \multirow[b]{3}{*}{ Example } & \multirow{3}{*}{$\begin{array}{c}\text { Date } \\
\text { (1979) }\end{array}$} & \multirow{3}{*}{$\begin{array}{c}\text { Time } \\
\text { (GMT) }\end{array}$} & \multirow{3}{*}{$\begin{array}{l}\text { Lat. } \\
\left({ }^{\circ} \mathrm{S}\right)\end{array}$} & \multirow{3}{*}{$\begin{array}{l}\text { Lon. } \\
\left({ }^{\circ} \mathrm{E}\right)\end{array}$} & \multicolumn{12}{|c|}{ Great circle heading (HD) and distance (DS) to: } \\
\hline & & & & & \multicolumn{2}{|c|}{$\begin{array}{c}\text { Japan } \\
\text { (1) }\end{array}$} & \multicolumn{2}{|c|}{$\begin{array}{l}\text { Norway } \\
\text { (2) }\end{array}$} & \multicolumn{2}{|c|}{$\begin{array}{l}\text { Liberia } \\
\text { (3) }\end{array}$} & \multicolumn{2}{|c|}{$\begin{array}{c}\text { Hawaii } \\
\text { (4) }\end{array}$} & \multicolumn{2}{|c|}{$\begin{array}{c}\text { La- } \\
\text { Reunion } \\
\text { (6) }\end{array}$} & \multicolumn{2}{|c|}{$\begin{array}{l}\text { Argentina } \\
\text { (7) }\end{array}$} \\
\hline & & & & & HD & DS & HD & DS & HD & DS & HD & DS & HD & DS & HD & DS \\
\hline A & 18 Jun & 0818 & 13.9 & 58.6 & 58 & 66 & 341 & 60 & 271 & 69 & 46 & 130 & 185 & 35 & 227 & 124 \\
\hline B & $27 \mathrm{Jun}$ & 0524 & 14.9 & 67.5 & 58 & 59 & 338 & 63 & 273 & 77 & 52 & 1.23 & 199 & 38 & 225 & 131 \\
\hline
\end{tabular}

Hawaii are more consistent. Furthermore, wind speeds estirnated with Hawaii are consistently lower than those estimated without it.

If local Omega signals are available, long-path propagation may be detected by examining the sign of the phase changes as the signal in question passes through either terminator. However, the lack of local Omega in the aircraft ODW system requires another approach. Among the simplest remedies are the omission of all Omega signals originating $>100^{\circ}$ to the east of the sonde, or the calculation of wind profiles with and without the suspect signal.

A. graphical solution to this problem takes advantage of differences in hyperbolic geometry for stations east and west of an ODW. The different orientation and spacing of the hyperbolic phase lanes give rise to characteristic "families" of hyperbolic phase curves for the eastward and westward stations. By examining the time behavior of phase for hyperbolic pairs known to be propagating normally, stations propagating longpath can be identified. As an example, we present a test drop from Patrick Air Force Base, Florida. Figure 1 shows the great circle geometry for the drop. Note that Liberia and LaReunion lie on nearly the same azimuth, and that Hawaii and LaReunion are on nearly opposite ones. Based on the discussion above and Fig. 1, LaReunion must be considered the most likely candidate for long-path propagation. The central station of Norway is selected as the common station and the following hyperbolic (difference) phases are calculated: Norway-North Dakota and Norway-Hawaii (the westward stations); Norway-Liberia and
Norway-LaReunion (the eastward stations). These differences are shown in Figs. 5 and 6. The NorwayLeReunion curve appears to be in the wrong figure; it looks like the curves for the westward stations, Hawaii and North Dakota. We conclude, then, that LaReunion is propagating long-path. HRD postprocessing uses this technique to identify long-path signals, while in the GWE the simple rule of eliminating all signals originating $>100^{\circ}$ to the east was adopted. In some areas, the Indian Ocean, for example, such a practice often renders the remaining sonde-transmitter geometry nearly useless. The addition of the Australian station in 1981 now provides better geometry over much of the Pacific Ocean, but the southern Indian Ocean still remains a marginal area for Omega windfinding.

\section{Internal factors-comparison of phase-smoothing algorithms}

In the normal use of ODWs, the winds are unknown, which makes accurate evaluations of wind error difficult or impossible. In this study, Omega wind errors are measured directly by using motionless sondes. On 4 August 1982 the WP-3D aircraft of the NOAA Office of Aircraft Operations (OAO) flew an experiment during which Omega signals were received from a stationary sonde on Key Largo, Florida. The aircraft flew $\mathrm{L}$ patterns (Fig. 7 ) and received adequate Omega for windfinding for about $95 \mathrm{~min}$. Actual Omega wind errors during this experiment are given by the computed wind speed. With this data set we

TABLE 3. $850 \mathrm{mb}$ wind vectors, uncertainties (WU) for Examples A and B (Table 2), computed for various Omega station combinations. Units are degrees and meters per second. Station numbers and names correspond as follows: 1-Japan, 2-Norway, 3-Liberia, 4-Hawaii, 5North Dakota, 6-LaReunion, 7-Argentina.

\begin{tabular}{clcccccc}
\hline \hline & & \multicolumn{5}{c}{ Combinations } \\
\cline { 3 - 7 } Example & Quantity & $2,3,6$ & $1,2,3,6$ & $2,3,7$ & $3,4,7$ & $3,4,6$ & $2,3,4,6,7$ \\
\hline \multirow{2}{*}{ A } & Direction/speed & $266 / 22.5$ & $266 / 21.5$ & $259 / 30.2$ & $279 / 7.3$ & $311 / 13.6$ & $284 / 9.3$ \\
& WU & 3.6 & 2.3 & 13.6 & 6.2 & 1.5 & 1.2 \\
\multirow{2}{*}{ B } & Direction/speed & $255 / 19.9$ & $255 / 17.0$ & $255 / 20.0$ & $026 / 2.7$ & $355 / 8.2$ & $150 / 3.0$ \\
& WU & 9.5 & 1.5 & 9.5 & 4.4 & 1.5 & 1.1 \\
\hline
\end{tabular}




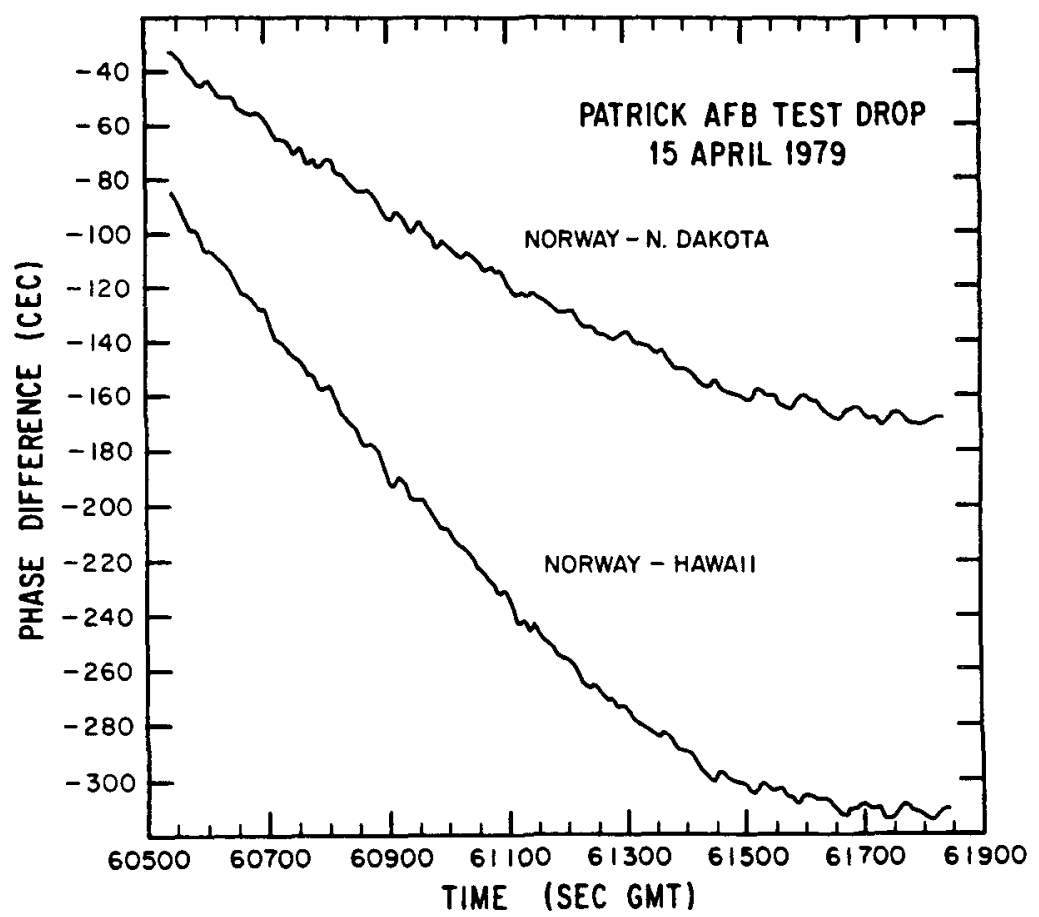

FIG. 5. Hyperbolic phases (with Norway as the common station) for transmitters west of Patrick AFB test drop on 15 April 1979.

can investigate the internal error factors of aircraft ment, the effects of varying geometry and noise levels acceleration, noise and transmitter-sonde geometry. can be simulated by computing winds with many Although only one sonde is involved in this experi- different station combinations and by using raw and

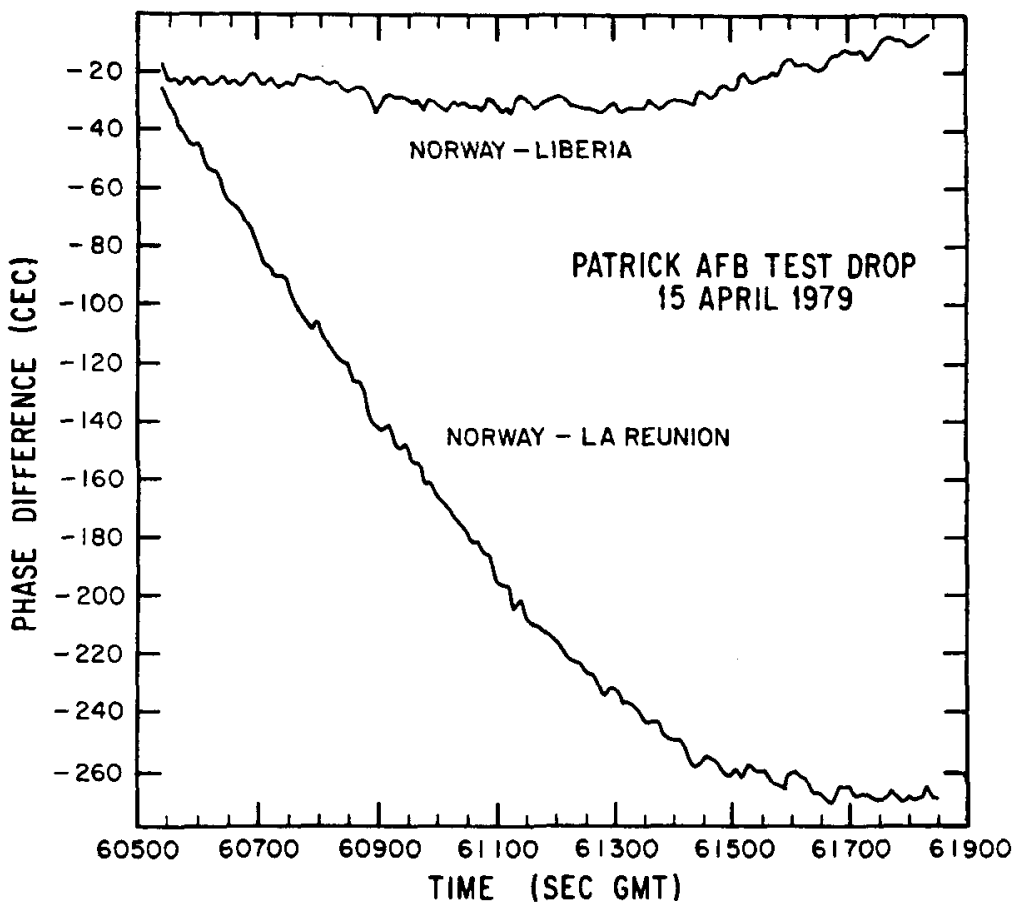

FIG. 6. Hyperbolic phases (with Norway as the common station) for transmitters east of Patrick AFB test drop on 15 April 1979. 


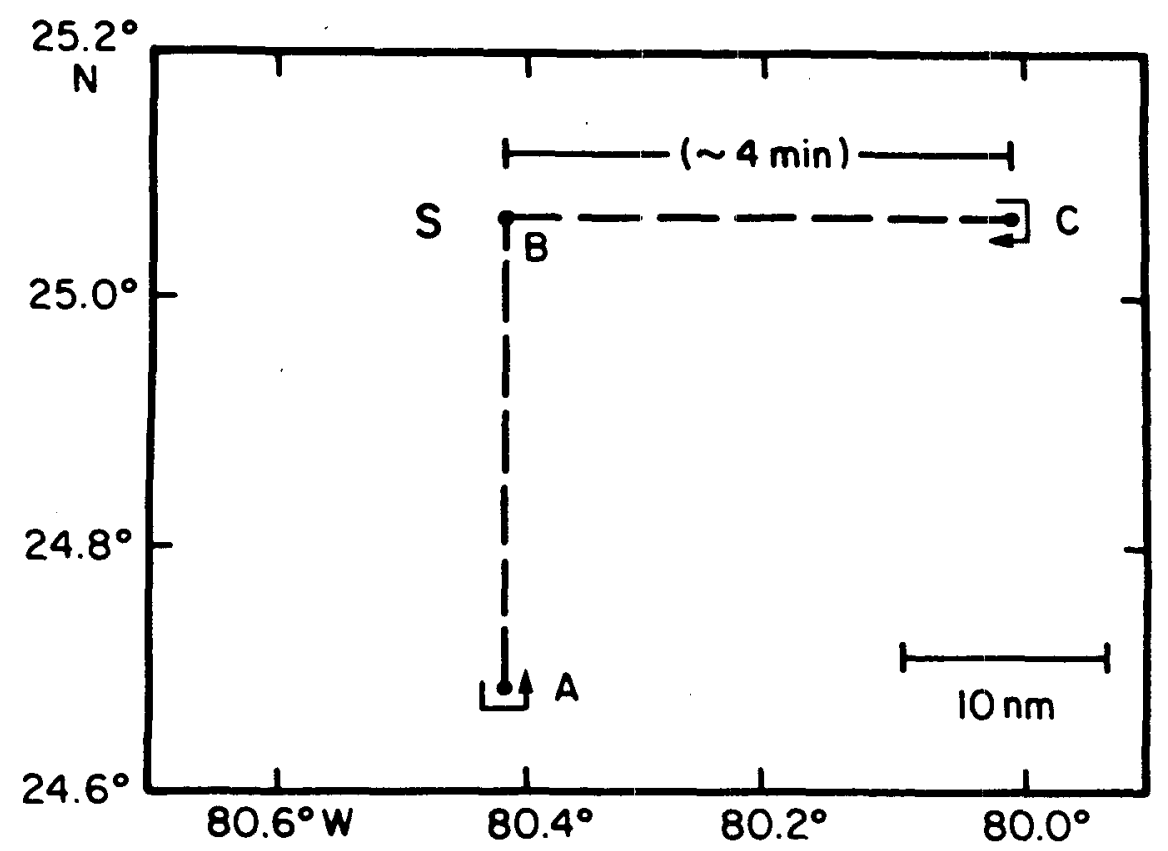

FIG. 7. Flight track for 4 August 1982. Position of the stationary sonde is marked by the "S." Turn types are identified by letter.

edited Omega signals. The geometry for this experiment is nearly identical to that shown in Fig. 1.

As Omega signals were received during the flight, the on-board computer calculated winds for real-time inspection. During postprocessing at HRD, the phase data were examined for noise spikes and other problems that typically degrade the quality of on-board wind estimates. Noise spikes in the real-time (raw) phases were subjectively removed to produce an "edited" phase data set. Such editing is standard procedure in ODW postprocessing; it improves the accuracy of winds computed from raw Omega on board (Franklin, 1983). Figure 8 shows examples of typical raw and edited Omega data from the flight. The rapid changes in slope identify the aircraft turns which are labeled by letters that correspond to the turn points in Fig. 7. Notice that the worst Omega reception tends to occur while the aircraft turns. This partial signal loss is caused by shadowing of the Omega antenna: as the roll angle of the aircraft increases to execute a turn, the antenna (mounted on the underside of the plane) generally finds itself behind the aircraft relative to the sonde; this temporarily reduces signal strength. As a result of shadowing, one expects real-time Omega-derived wind accuracy to be low during turns. It is demonstrated below that winds computed with some phase-smoothing algorithms are less accurate during turns even after the effects of shadowing have been removed.

Omega winds are usually computed from as many Ornega stations as are available (except in real-time, when no more than four may be used). So that the results of this study would be applicable to areas outside south -Florida, winds were computed using 12 station combinations in an attempt to simulate the geometries of other regions. This set of combinations is only a subset of all possible geometries, but it was chosen to include a wide range of reasonable choices. Geometries that would produce extremely large wind errors were excluded. Each of the three phase-smoothing algorithms (quadratic, filter and cubic-spline) was used with each combination for raw (W) and edited (E) phases, so that, in all 72 , wind time series (profiles) were produced. For each of the 72 profiles, the following instantaneous quantities were calculated at $10 \mathrm{~s}$ intervals: the actual wind error $\mathrm{WE}=\left(U^{2}+V^{2}\right)^{0.5}$, where $U$ and $V$ are given by (10); the wind uncertainty WU $=\left[\operatorname{Tr}\left(\mathbf{F}^{T} \mathbf{E}^{-1} \mathbf{F}\right)^{-1} K\right]^{0.5}$; and the wind error ratio $R$ $=\mathrm{WE} / \mathrm{WU}$. In the discussion that follows, $\bar{X}$ denotes the profile (time) average of the quantity $X$ over the 95 min flight, and $\langle\bar{X}\rangle$ refers to an average of $\bar{X}$ over the 12 station combinations. For example, $\overline{W E}(Q \bar{U} A D, 2345 \mathrm{~W})$ refers to the mean wind error for the raw phase data, computed using the quadratic windfinder with stations 2, 3, 4 and 5 (Norway, Liberia, Hawaii and N. Dakota; see Fig. 1).

\section{a. Accuracy during aircraft accelerations}

The effect of aircraft accelerations is most pronounced with on-board unprocessed Omega. Since only the quadratic smoothing algorithm is available onboard, the effects of aircraft turns are described primarily in terms of quadratic smoothing. 


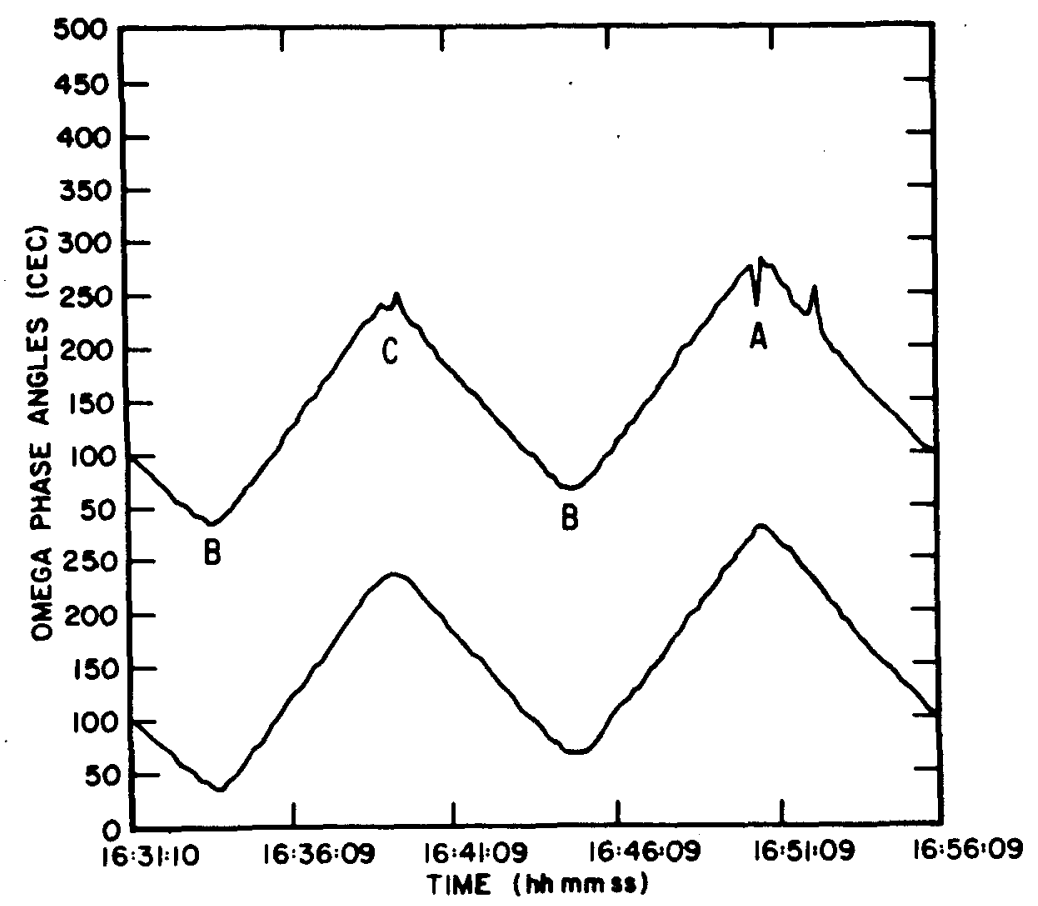

FIG. 8. Raw (top) and edited (bottom) Omega signals from the Norway station for part of the 1982 mission. Turn labeling corresponds to that in Fig. 7.

Figure 9 shows wind uncertainty WU(QUAD, $234578 \mathrm{E}$ ) plotted against time for a portion of the experiment. During this time the NOAA aircraft made four turns, identifed by the dashed arrows. In the neighborhood of each turn, WU increases from about $1.2 \mathrm{~m} \mathrm{~s}^{-1}$ during periods of straight and level motion (legs) to well over $3 \mathrm{~m} \mathrm{~s}^{-1}$. These intervals of high WU are much larger than the length of the turns, since each wind estimate is obtained from a window of phase data. For the quadratic and cubicspline smoothers, then, a wind estimate for time $t_{0}$ is defined to be part of the turn sample if $t_{0}$ is within $105 \mathrm{~s}$ of the center of an actual aircraft turn. For the $4 \mathrm{~min}$ low-pass filter, this limit is $135 \mathrm{~s}$.

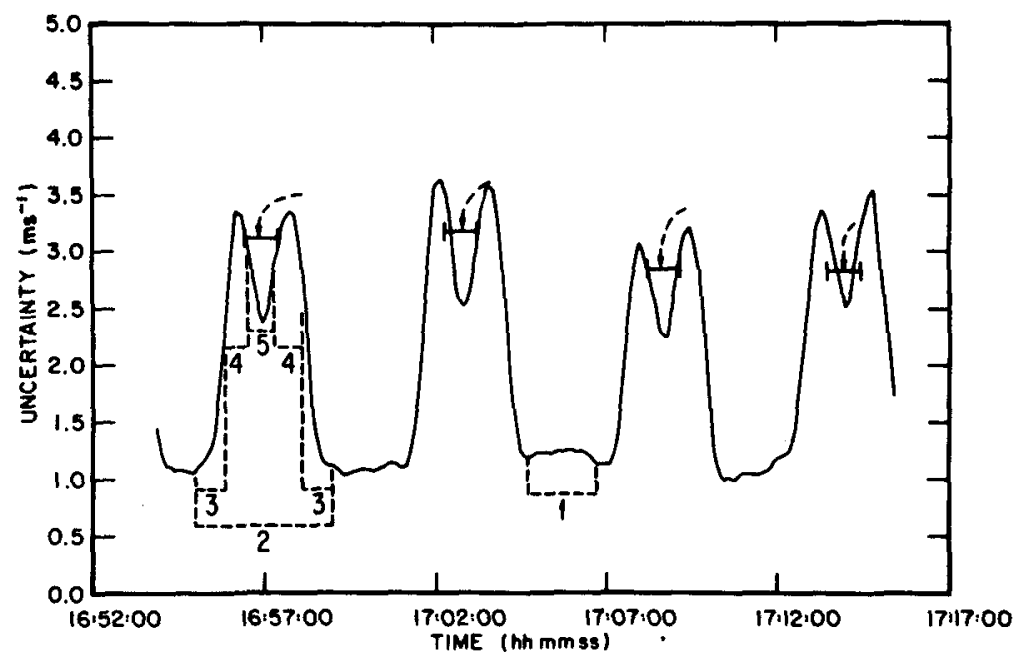

FIG. 9. Wind uncertainty for a portion of wind profile (QUAD, 234578E) (see text). Aircraft accelerations (turns) are identified by the arrows and brackets. Stratification of points by position relative to aircraft turns is indicated by number: $1=\operatorname{leg}, 2=$ turn, $3=$ turn edge, $4=$ turn peak, $5=$ turn center. 
TABLE 4. Error statistics for (QUAD, 234578E).

\begin{tabular}{lccccc}
\hline \hline & $\begin{array}{c}\text { Sample } \\
\text { size }\end{array}$ & $\begin{array}{c}\text { Independent } \\
\text { sample size }\end{array}$ & $\begin{array}{c}\overline{\mathrm{WE}} \\
\left(\mathrm{m} \mathrm{s}^{-1}\right)\end{array}$ & $\begin{array}{c}\overline{\mathrm{WU}} \\
\left(\mathrm{m} \mathrm{s}^{-1}\right)\end{array}$ & $\bar{R}$ \\
\hline Legs & 228 & 107 & 1.93 & 1.19 & 1.63 \\
Turns & 351 & 165 & 2.51 & 2.65 & 1.06 \\
\hline$-5 d g e s$ & 136 & 64 & 2.49 & 1.98 & 1.39 \\
$\quad$ Peaks & 140 & 66 & 2.44 & 3.29 & 0.75 \\
$\quad$ Centers & 75 & 35 & 2.66 & 2.69 & 1.01 \\
\hline
\end{tabular}

One aspect of Fig. 9 is the double peak of WU for each turn. A natural question is whether WE behaves in the same fashion. To address this question, turn points are stratified into subgroups based on the WU curve in Fig. 9 and are referred to as edge, peak and center points. The definitions of these groups are given schematically in the figure.

Table 4 presents mean wind uncertainty and mean wind error for the wind profile (QUAD, 234578E). The table gives two sample sizes for each group; the first of these is the actual count of points in the sample. Each of these is not an independent wind estimate, however, since the 19-point phase window centered on $t_{0}$ is nearly identical to the window centered on $t_{0}+10$. An equivalent number of independent estimates can be made using the method described by Shapiro (1984). The sequence of values WE(QUAD, 234578E) was evaluated for serial correlation using this procedure; the equivalent independent sample was found to be $47 \%$ as large as the original sample. That nearly every second wind estimate is independent with the quadratic algorithm indicates that winds from quadratic smoothing are extremely dependent on phase points at the edges of the $3 \mathrm{~min}$ window.

Table 4 shows that WU, which depends on the mean least-squares residual and not on the location of the residuals within the window, underpredicts wind errors about $90 \mathrm{~s}$ before and after the actual occurrence of a turn. The table also indicates that turns will degrade ODW wind estimates over a full 3 min $(75 \mathrm{mb})$ interval. (The difference between wind errors in and out of turns can be shown to be statistically significant.) It should be remembered that we are considering edited data here, from which the effects of shadowing have been removed; the poorer performance of quadratic smoothing in turns must then reflect the quadratic's assumption of constant acceleration.

Now consider Table 5, which presents $\overline{\mathrm{WE}}$ and $\overline{\mathrm{W}} \overline{\mathrm{U}}$ for all the wind profiles computed from the 1982 experiment. For now, consider only the columns

TABLE 5. Sonde-mean wind errors and wind uncertainties for 1982 research flight (stationary sonde with aircraft flying "L" pattern). Wind error is given first; all values are in $\mathrm{m} \mathrm{s}^{-1}$. Station names and numbers correspond as shown in Fig. 1 .

\begin{tabular}{|c|c|c|c|c|c|c|c|c|c|c|c|c|}
\hline \multirow[b]{3}{*}{ Stations } & \multicolumn{6}{|c|}{ Legs } & \multicolumn{6}{|c|}{ Turns } \\
\hline & \multicolumn{3}{|c|}{ Edited Omega } & \multicolumn{3}{|c|}{ Raw Omega } & \multicolumn{3}{|c|}{ Edited Omega } & \multicolumn{3}{|c|}{ Raw Omega } \\
\hline & Quad & Filter & Spline & Quad & Filter & Spline & Quad & Filter & Spline & Quad & Filter & Spline \\
\hline & 1.93 & 1.83 & 1.39 & 2.02 & 2.27 & 1.31 & 2.51 & 2.31 & 1.40 & 2.91 & 2.71 & 1.30 \\
\hline \multirow[t]{2}{*}{2345.78} & 1.19 & 1.94 & 0.95 & 1.55 & 1.99 & 0.95 & 2.65 & 3.42 & 1.07 & 3.64 & 4.29 & 1.12 \\
\hline & 1.99 & 1.89 & 1.42 & 2.15 & $2.57^{\circ}$ & 1.63 & 2.39 & 2.14 & 1.46 & 3.39 & 3.27 & 1.42 \\
\hline \multirow[t]{2}{*}{2345.7} & 1.35 & 2.22 & 1.11 & 1.78 & 2.29 & 1.10 & 3.02 & 3.88 & 1.22 & 4.19 & 4.95 & 1.22 \\
\hline & 2.25 & 2.14 & 1.56 & 2.77 & 2.80 & 1.67 & 2.62 & 2.31 & $1.5 \mathrm{t}$ & 3.90 & 3.71 & 1.46 \\
\hline \multirow[t]{2}{*}{2347} & 1.59 & 2.49 & 1.32 & 2.53 & 2.64 & 1.30 & 3.33 & 4.25 & 1.45 & 4.78 & 5.54 & 1.46 \\
\hline & 2.29 & 2.23 & 1.64 & 2.50 & 2.79 & 2.01 & 2.69 & 2.51 & 1.60 & 4.06 & 3.84 & 1.67 \\
\hline \multirow[t]{2}{*}{2457} & 1.67 & 2.71 & 1.57 & 2.14 & 2.86 & 1.52 & 3.66 & 4.68 & 1.76 & 5.11 & 6.00 & 1.76 \\
\hline & 2.59 & 2.53 & 2.15 & 2.71 & 3.06 & 2.06 & 3.34 & 3.22 & 1.94 & 4.01 & 3.87 & 1.83 \\
\hline \multirow[t]{2}{*}{2345} & 1.97 & 3.35 & 1.92 & 2.57 & 3.62 & 2.00 & 4.60 & 5.94 & 1.98 & $6.38^{\circ}$ & 7.62 & 2.15 \\
\hline & 2.98 & 2.55 & 1.96 & 3.55 & 3.48 & 2.09 & 3.48 & 3.22 & 1.98 & 4.87 & 4.69 & 1.94 \\
\hline \multirow[t]{2}{*}{2378} & 1.82 & 2.73 & 1.39 & 2.55 & 2.97 & 1.39 & 3.58 & 4.65 & 1.55 & 5.47 & 6.19 & 1.65 \\
\hline & 2.55 & 2.40 & 1.63 & 2.98 & 3.01 & 2.01 & 2.87 & 2.61 & 1.61 & 4.78 & 4.39 & 1.67 \\
\hline \multirow[t]{2}{*}{247} & 1.86 & 2.94 & 1.68 & 2.43 & 3.37 & 1.64 & 3.92 & 4.99 & 1.89 & 5.78 & 6.66 & 1.91 \\
\hline & 2.99 & 2.55 & 2.33 & 4.04 & 3.42 & 2.41 & 3.68 & 3.19 & 2.16 & 6.04 & 5.58 & 2.05 \\
\hline \multirow[t]{2}{*}{257} & 2.39 & 4.00 & 2.36 & 4.26 & 4.59 & 2.29 & 5.43 & 6.98 & 2.74 & 8.37 & 9.71 & 2.79 \\
\hline & 3.48 & 2.62 & 3.26 & 3.80 & 3.64 & 3.06 & 5.40 & 4.35 & 2.99 & 6.21 & 5.61 & 2.79 \\
\hline \multirow[t]{2}{*}{245} & 4.74 & 8.79 & 5.12 & 6.55 & 9.92 & 5.25 & 12.68 & 16.45 & 5.25 & 16.63 & 19.39 & 5.41 \\
\hline & 3.61 & 3.23 & 2.73 & 4.05 & 3.77 & 2.40 & 4.24 & 3.95 & 2.34 & 6.24 & 5.67 & 2.28 \\
\hline \multirow[t]{2}{*}{234} & 2.66 & 4.10 & 2.53 & 4.02 & 4.74 & 2.65 & 5.34 & 6.78 & 2.73 & 8.66 & 9.95 & 3.12 \\
\hline & 3.51 & 3.01 & 2.29 & 4.35 & 3.81 & 2.47 & 4.40 & 4.10 & 2.26 & 6.00 & 5.56 & 2.27 \\
\hline \multirow[t]{2}{*}{278} & 2.35 & 3.48 & 1.95 & 3.41 & 3.92 & 1.94 & 4.59 & 5.77 & 2.14 & 7.00 & 7.72 & 2.21 \\
\hline & 4.95 & 4.42 & 3.15 & 5.56 & 5.85 & 4.37 & 6.38 & 5.53 & 3.04 & 9.24 & 8.61 & 3.63 \\
\hline 458 & 5.63 & 9.54 & 5.57 & 7.52 & 10.09 & 5.41 & 13.12 & 16.88 & 6.78 & 17.17 & 19.46 & 6.50 \\
\hline$\langle\mathrm{WE}\rangle$ & 2.93 & 2.62 & 2.13 & 3.37 & 3.37 & 2.29 & 3.67 & 3.29 & 2.02 & 5.14 & 4.79 & 2.03 \\
\hline$\langle\mathbf{W U}\rangle$ & 2.44 & 4.02 & 2.29 & 3.44 & 4.42 & 2.29 & 5.49 & 7.06 & 2.55 & 7.77 & 8.96 & 2.61 \\
\hline
\end{tabular}


for quadratic smoothing. For every geometry, $\overline{\mathrm{WE}}$ for turns is higher than $\overline{W E}$ for legs, for both edited and raw Omega. When we average over geometry as well as time, $\langle\overline{\mathrm{WE}}\rangle$ for turns exceeds $\langle\overline{\mathrm{WE}}\rangle$ for legs by $25 \%$ (3.7 versus 2.9) for edited Omega, while for raw Omega the difference is $53 \%$ (5.1 versus 3.4). The lower turn errors for edited Omega reflect the correction of low signal quality caused by shadowing during turns.

Phase editing is one way to improve wind accuracy in turns; another way is to use a different smoothing algorithm in the postprocessing. From Table 5, one sees that the spline is not influenced by the presence of turns. In the "best case" of (SPL, 234578E), WE for turns and legs are both about 1.4. The spline is the only algorithm (of the three studied here) in which wind accuracy is not reduced in turns. One reason for this behavior is the absence of the constant acceleration implication of the quadratic. Another factor, though, may be the continuity restrictions of the spline, by which phase data minutes away can affect a wind estimate. These restrictions would tend to blur the distinction between the turn and leg samples; it may still be true that spline winds are less accurate when turns are in the flight pattern, although the data collected for this study will not help to answer this question.

When we compare turn accuracy using the spline to the two other algorithms, we find significant differences. For best-case geometry $(234578 \mathrm{E})$, mean error for the spline is $1.4 \mathrm{~m} \mathrm{~s}^{-1}$ as noted above, compared with 2.3 and $2.5 \mathrm{~m} \mathrm{~s}^{-1}$ for the filter and quadratic (Table 5). A modified $t$-test on the equality of these means gives $p$-values of less than 0.01 for both the quadratic and the filter smoothers. This $40 \%$ reduction of error with the spline remains approximately the same when averaged over geometry. The spline, then, should be the smoothing algorithm of choice when turns are an important part of the flight track.

\section{b. Accuracy during straight and level flight}

Figure 10 shows wind error and uncertainty for a portion of the $(234578 \mathrm{E})$ wind profiles for each algorithm. Quadratic wind estimates, with their high dependence on window end points, vary most rapidly. The plot of WE for the filtered phases looks much like a smoothed version of the quadratic. The cubicspline profile is nothing like the other two, however, with slowly changing errors and uncertainties that bear little or no relationship to the turns.

These scales of variability are also indicated by the calculations of independent sample size for the three windfinders. Recall that the sample size for quadratic wind estimates had to be reduced by only a factor of 2 to account for the serial dependence of the estimates. For the low-pass filter, the equivalent independent

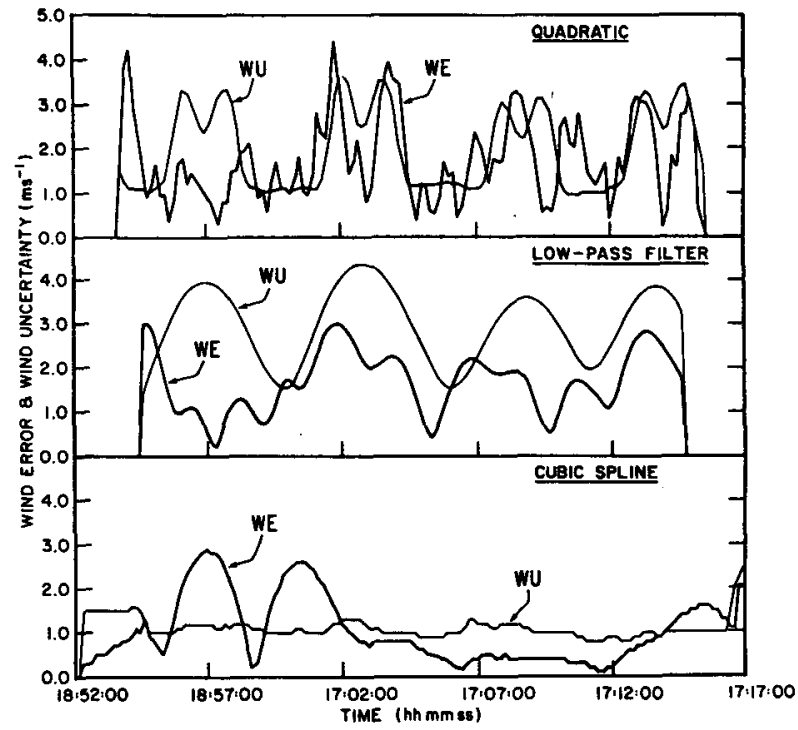

FIG. 10. Predicted wind uncertainty (WU-thin line) and measured wind error (WE-thick line) for a portion of the profiles (234578E) for the three phase-smoothing algorithms.

sample was found to be $16 \%$ as large as the full sample, while for the spline, only $11 \%$. This outcome is not surprising, since both filter and spline wind estimates are influenced by phase data minutes away, while quadratic estimates are primarily determined by a few points at the edges of the $3 \mathrm{~min}$ window.

We return to Table 5 , to compare algorithm accuracy during straight and level flight (legs). Looking at the left-hand side of the table, we see that for both raw and edited Omega, spline smoothing produces the smallest errors. For best-case edited signals, spline mean error is $24 \%$ lower than the low-pass filter and $28 \%$ lower than the quadratic, with means of $1.4,1.8$ and $1.9 \mathrm{~m} \mathrm{~s}^{-1}$, respectively. The modified $t$-test on the equality of the filter and spline means gives a $p$ value of 0.15 , a value not statistically significant due to the small independent sample sizes, but suggestive nonetheless. For the quadratic and spline means, the $p$-value is lower at 0.01 . When we average over geometry, errors with spline smoothing are $19 \%$ and $27 \%$ lower than with low-pass filtering and quadratic smoothing. The cubic spline has the lowest mean wind error in 23 of the 24 "contests" on the lefthand side of Table 5. Although statistical significance is not quite achieved over the other algorithms, it should be clear that the spline is the superior smoothing algorithm for legs as well as turns.

It should be pointed out that although the spline algorithm allows the calculation of winds from the first phase point to the last, winds computed in the first and last $90 \mathrm{~s}$ of each profile were not included in any of the means in Table 5. These wind estimates were omitted to keep the samples for the three methods roughly equivalent. As one might expect, 
wind accuracy at the edges of the spline is not as high as in the interior. For the case of (SPL, 234578E), wind error in the edges is $3.0 \mathrm{~m} \mathrm{~s}^{-1}$, compared with $1.4 \mathrm{~m} \mathrm{~s}^{-1}$. over the remainder of the profile. Until this effect has been investigated in more detail, much care should be taken in the use of spline winds at the beginning and end of drops.

\section{c. Resolution of wind shear}

Although the spline is more accurate than the quadratic or filter for the stationary test sonde, one might be concerned about the spline's resolution, and hence, its suitability for soundings with significant wind shear. To examine the question of resolution, a time series of simulated Omega signals was created. Using (8), noise-free phase rates corresponding to selected values of $U$ and $V$ were computed to form a 20 min "synthetic" sounding. For simplicity, phases were created for only three stations (Norway, North Dakota and Argentina). The winds chosen for the synthetic sounding were taken from rawindsonde data for Dodge City, Kansas, at 0000 GMT 6 December 1978 and feature a distinct frontal zone with a wind shear of $2.4 \times 10^{-2} \mathrm{~s}^{-1}$ between 725 and 750 mb (Fig. 11). A shear of similar magnitude is also observed near $650 \mathrm{mb}$. "Observed" values of $U$ and $V$ at $20 \mathrm{~s}(\sim 10 \mathrm{mb})$ intervals were obtained by linear interpolation between the points in Fig. 11.

Wind errors for the three windfinders are also shown in Fig. 11. Errors increase dramatically in the frontal zone; none of the algorithms can accurately resolve such a wind shift. Wind errors are also large above the frontal zone in the second region of highest shear. Over most of the sounding, however, errors are generally $<2 \mathrm{~m} \mathrm{~s}^{-1}$.

The spline is clearly the weakest of the three windfinders in terms of wind-shear resolution. Average error for the spline with this sounding is $1.9 \mathrm{~m} \mathrm{~s}^{-1}$, compared with average errors of 1.0 and $0.9 \mathrm{~m} \mathrm{~s}^{-1}$ for the filter and quadratic algorithms. In the two regions of highest shear, the spline has by far the highest errors, and below the frontal zone, a brief veering of the wind to the northeast is picked up by the quadratic and filter, but not by the spline.

One must be very careful in drawing conclusions from this experiment, however. The poor performance of the spline in resolving the fine structure of the wind field does not imply that the quadratic or filter algorithms would yield more accurate winds in a real sounding. The continuity restrictions at the nodal points of the spline, which are responsible for its relative lack of resolution, are also responsible for the spline's ability to extract accurate phase rates from real (i.e., noisy) Omega (as demonstrated by the stationary sonde experiment). There is an important trade-off between resolving power and sensitivity to noise. Through its continuity restrictions, the spline increases its effective sample of phase points for the cubic fit, reducing phase variance and the effects of

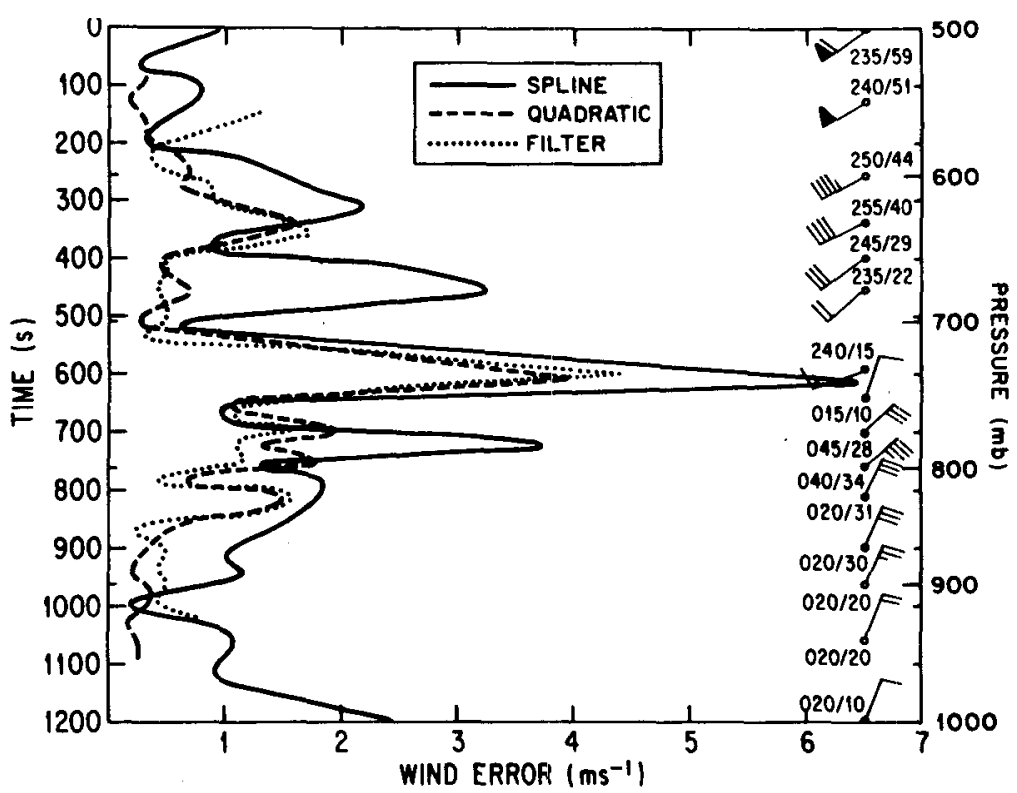

FIG. 11. Wind profile of the synthetic sounding with associated wind errors for the three phase-smoothing algorithms. Rawinsonde data, from which synthetic Omega signals were derived, appear along the right side of the figure. Values of wind direction and speed (in knots) appear next to each plotted wind vector. Errors for each smoothing algorithm in reproducing this sounding are given on the left side of the diagram. 
noise, but in doing so it ignores "local" changes in phase rate that represent regions of high shear. The quadratic, on the other hand, examines only $3 \mathrm{~min}$ of data at a time. This makes the quadratic more responsive to smaller scale changes in phase rate, but also makes it susceptible to unrepresentative phase measurements.

The current accuracy of Omega phase measurement dictates that windfinding algorithms primarily must be able to handle noise. This can be demonstrated by adding artificial noise to the synthetic sounding. For simplicity, a crude noise model consisting of two components was employed. A random "error," taken from a Gaussian distribution with zero mean and standard deviation of $1.5 \mathrm{cec}$ was added to every synthetic phase point. A second error, taken from a uniform distribution on the interval $(0,4) \mathrm{cec}$, was randomly added to $20 \%$ of the phase points. (In the stationary sonde experiment, $60 \%$ of the subjective phase edits corrected the phase downward. This bias toward positive phase errors is also observed in HRDs raw phase data for 1982s Hurricane Debby.) This model for noise produced phase time series with variances [given by (12)] of about $2.5 \mathrm{cec}^{2}$, a noise level found in Omega signals of above average quality. When the artificial phase errors were added to the sounding, mean wind error with the quadratic increased $139 \%$ to $2.2 \mathrm{~m} \mathrm{~s}^{-1}$. With the filter, mean error increased $87 \%$ to 1.9 , while with the spline, mean error increased only $18 \%$ to $2.2 \mathrm{~m} \mathrm{~s}^{-1}$.

When flight tracks involve turns, it is clear that the cubic spline can provide the most accurate wind estimates. In cases where there are no turns, however, the situation is more complicated, due to the tradeoff between noise sensitivity and resolution. In cases of very low phase variance (about $1.5 \mathrm{cec}^{2}$ or less), the quadratic or filter could be used to maximize vertical resolution. When phase variances are in the 2-3 $\operatorname{cec}^{2}$ range, sounding-mean wind error is probably about the same for the three algorithms; in these cases, we feel that the spurious noise related wind shifts in a quadratic or filter-derived sounding are less desirable than the straightforward smoothing errors of the spline. For higher phase variances, the spline is the only acceptable algorithm. We recommend, then, that cubic-spline smoothing be used for nearly all ODW postprocessing.

\section{d. The effect of postprocessing}

The accuracy of operational (on-board) ODW wind estimates is limited largely by two factors: the availability of quadratic phase smoothing only, and by the presence of noise that can only be removed during postprocessing. By editing poor Omega and using the low-pass filter algorithm, Franklin (1983) observed changes in direction of at least $20^{\circ}$. or in speed of at least $5 \mathrm{~m} \mathrm{~s}^{-1}$ in $29 \%$ of the standard-level wind reports for the HRD flights around Hurricane Debby. With data from our stationary sonde, we can estimate the improvements to be expected from postprocessing by comparing WE(QUAD, W) to WE(SPL, E). Table 5 shows that in the geometric mean, postprocessing reduced mean wind error by $37 \%$ in the legs (3.4 to $2.1 \mathrm{~m} \mathrm{~s}^{-1}$ ) and by $57 \%$ in the turns (5.1 to $2.0 \mathrm{~m}$ $\left.\mathrm{s}^{-1}\right)$. For the individual geometries, error reductions were $14-47 \%$ in the legs and $52-67 \%$ in the turns.

These error reductions consider only the effects of editing and the use of the cubic-spline smoother. Table 5 shows that the particular combination of Omega stations used has a significant impact on wind accuracy. Often during postprocessing, wind profiles from several station combinations are examined before one is selected. In real time, there are no second chances. Further improvements in accuracy can be obtained by adding a fifth or sixth Omega signal to the postprocessed wind computation (no more than four may be used in real-time). $\overline{W E}(S P L, E)$ averaged over all four-station combinations is $1.8 \mathrm{~m} \mathrm{~s}^{-1}$, compared with $1.4 \mathrm{~m} \mathrm{~s}^{-1}$ for the five and six-station combinations. This additional $20-25 \%$ reduction in error would not occur if the four stations selected in real-time were nearly optimal, or if only three or four usable Omega signals were available. Nonetheless, in many cases this represents an additional advantage to postprocessing.

\section{$e$. The value of wind uncertainties}

Wind uncertainties (WU) or estimated wind errors, can be computed easily as part of the windfinding procedure. They depend upon two factors only: the quality of the sonde-station geometry, and the quality of the fit of the smoothed phases to the original phase data. They cannot take into account factors that are external to the dropwindsonde system, such as unsteady or anomalous propagation of the Omega signals. Even as estimates of internal errors they are of limited value; Fig. 10 hints that uncertainties are indicators only of mean wind accuracy over some period of time.

Fig. 12 is a scatter diagram of WU versus WE for every $10 \mathrm{~s}$ wind calculation of (QUAD, 234578E). The figure shows that knowledge of instantaneous wind uncertainty indicates almost nothing about the actual instantaneous wind error. Average values of WE and WU over the length of each wind profile are more informative. The $\overline{\mathrm{WE}}-\overline{\mathrm{WU}}$ pairs listed in Table 5 are plotted in Fig. 13 (for the legs only). Note first that the best profiles (those in the lower left of the figures) are produced by the cubic spline. Note also that for the raw phases, fitted curves relating $\overline{W E}$ to $\overline{W U}$ would be nearly identical for the three windfinding algorithms. The clustering of points close to the 


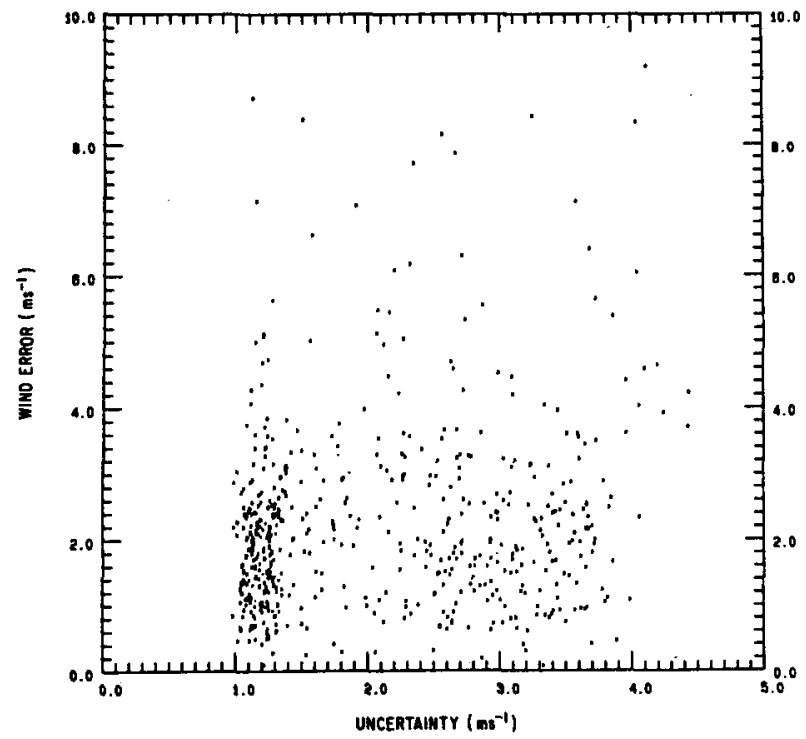

FIG. 12. Scatter diagram of wind error and uncertainty for (QUAD, 234578E). Each $10 \mathrm{~s}$ wind calculation is represented by an asterisk in the diagram.

$\overrightarrow{\mathrm{WE}}-\overline{\mathrm{WU}}$ line is satisfying, since it indicates that the wind uncertainties have been scaled fairly accurately. Winds computed from edited data show a division on the basis of algorithm, however, indicating that editing has had different effects on the three smoothing methods. Table 5 and Fig. 13 reveal that editing has little effect with spline smoothing, while its primary effect with the quadratic is to reduce uncertainty and with the filter to reduce actual error.

One feature of Fig. 13 is the nonlinearity of the $\overline{\mathrm{WE}}-\overline{\mathrm{WU}}$ relationship; i.e., the slope of this relationship is close to one for low uncertainty, but appears to approach zero as the uncertainty increases. This behavior is shown in a different way in Fig. 14. In this figure, for the 12 wind profiles (QUAD, E), each $10 \mathrm{~s}$ wind measurement is assigned to a class on the basis of its WU value. For example, all winds from the leg portions of (QUAD, 247E) with uncertainties between 1 and $2 \mathrm{~m} \mathrm{~s}^{-1}$ are represented by one point in Fig. 14. The means $\overline{\mathrm{WU}}$ and $\bar{R}(R=\mathrm{WE} / \mathrm{WU})$ for each class containing at least 15 points are plotted in the figure. Low uncertainties are seen to represent substantial underestimates of actual error, while high uncertainties tend to slightly overestimate the wind errors. This is not a behavior peculiar to the quadratic, since Fig. 15 shows a similar trend with the cubic spline. Some of this variation in $R$ seems to be due to editing. Figure 16 is the raw-Omega counterpart to Fig. 14, and although the dependence of $R$ on WU is reduced, there remains a behavior that cannot be explained by "human" interference.

Why the error ratio $R$ should depend upon the magnitude of the uncertainty is difficult to explain in terms of internal error sources. One is tempted to speculate that external sources of error may become evident when uncertainties are low, even though none of the propagation anomalies discussed in Section 3 seems likely to be responsible. If some propagation phenomenon is responsible for the behavior of the error ratio, Acheson showed remarkable insight when he suggested that "as the . . . noise problem diminishes with the use of high-power VLF transmitters, problems with propagation we have previously been able to ignore as being relatively small will likely become the more important [and] set the limit on windfinding performance." Another explanation, however, could lie in the assumption that the phaserate error matrix is proportional to the error matrix of the phases themselves. The scaling factor $K$ might, for example, depend on the signal-to-noise ratio of the incoming signals, rather than be constant, as Passi (1974) has heuristically argued. Values of $R$ are about 1.0 for uncertainty values of $2-3 \mathrm{~m} \mathrm{~s}^{-1}$ (Figs. 14-16); this is approximately the wind accuracy ex-
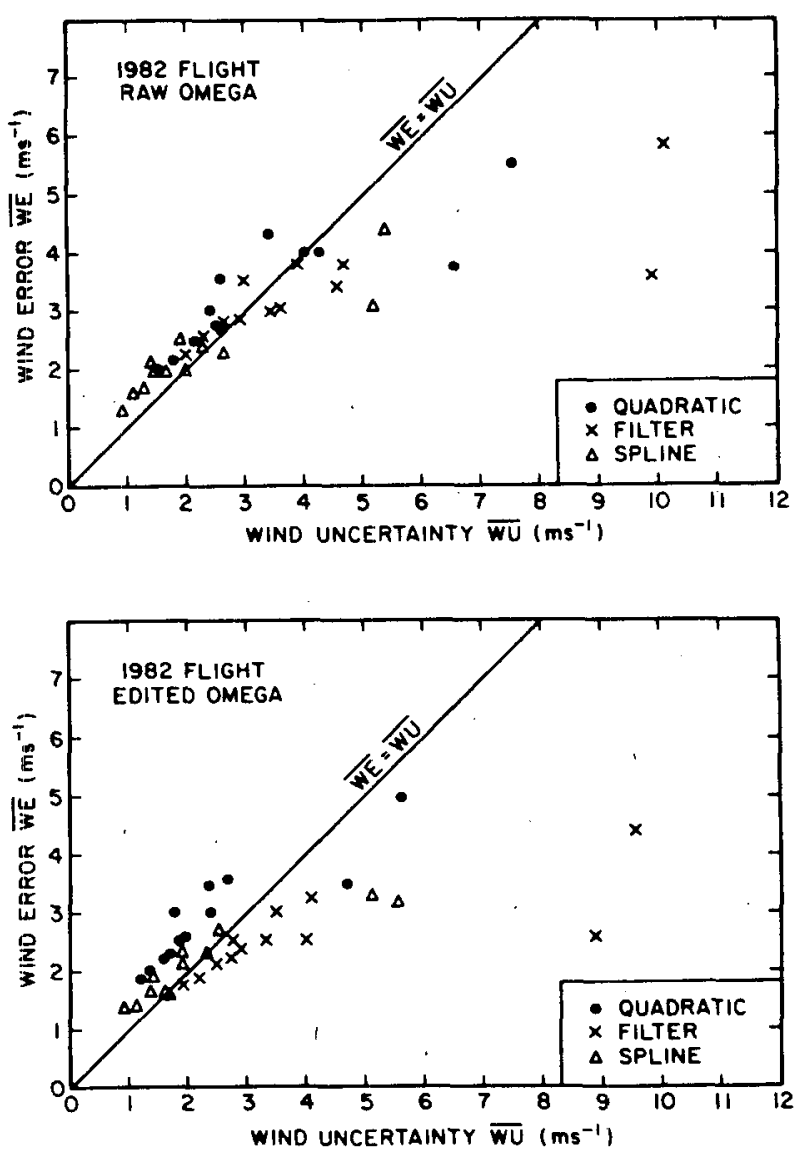

FIG. 13. Sonde-mean values of uncertainty and error taken from Table 5 for raw Omega (top) and edited Omega (bottom). 


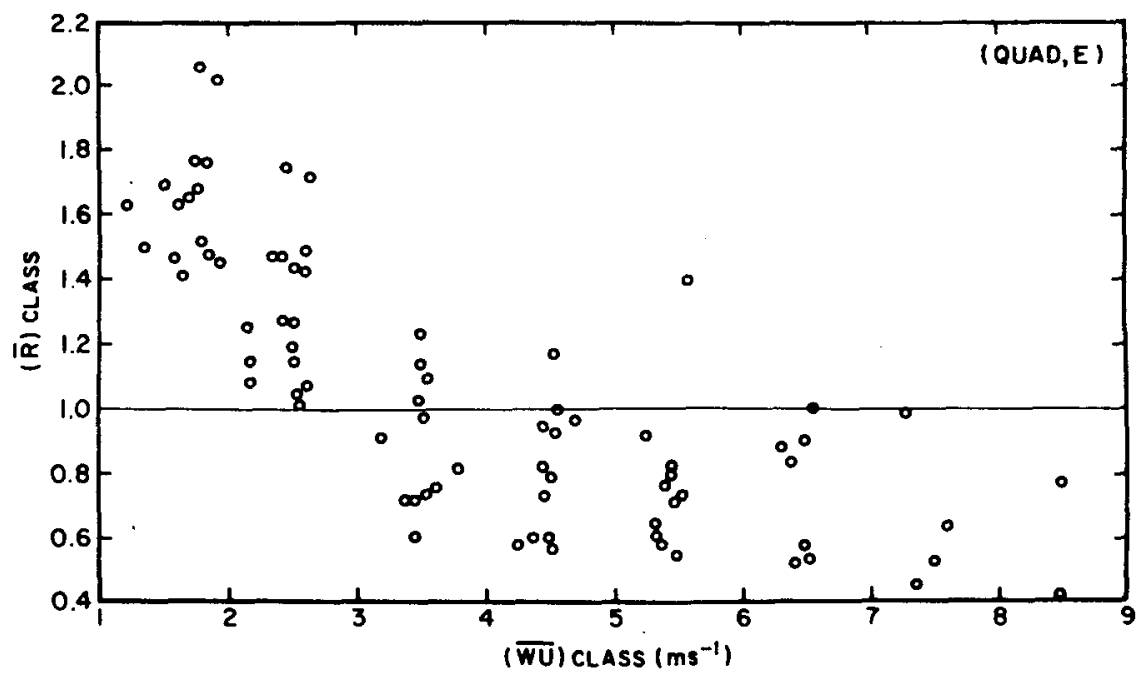

Fig. 14. Class-mean values (see text) of uncertainty and error ratio for the 12 (QUAD, E) profiles. Each plotted point represents a minimum of 15 wind measurements.

perienced during the 1978 test drops from which the scaling was determined.

\section{Summary and conclusions}

The errors accompanying Omega-derived wind estimates are of two main types: external error, which involve violations of the windfinding equations; and internal errors, which are due to variations in station geometry, signal quality, and base-station motion. Wind uncertainties, which are computed as part of the windfinding procedure, take into account only the internal effects.
The assessment of external sources of error generally depends on the availability of local Omega data (phase data not retransmitted from a moving sonde). These measurements show that errors from external sources range from $1 \mathrm{~m} \mathrm{~s}^{-1}$ for diurnal changes to 20-30 $\mathrm{m} \mathrm{s}^{-1}$ for sudden ionospheric disturbances. In the case of the aircraft dropsonde system, local Omega measurements are unavailable. Other, less precise methods of detection, along with the use of models derived from available local Omega, can be used to take these problems into account.

The effect of internal error sources is governed by the phase-smoothing algorithms that must be used to

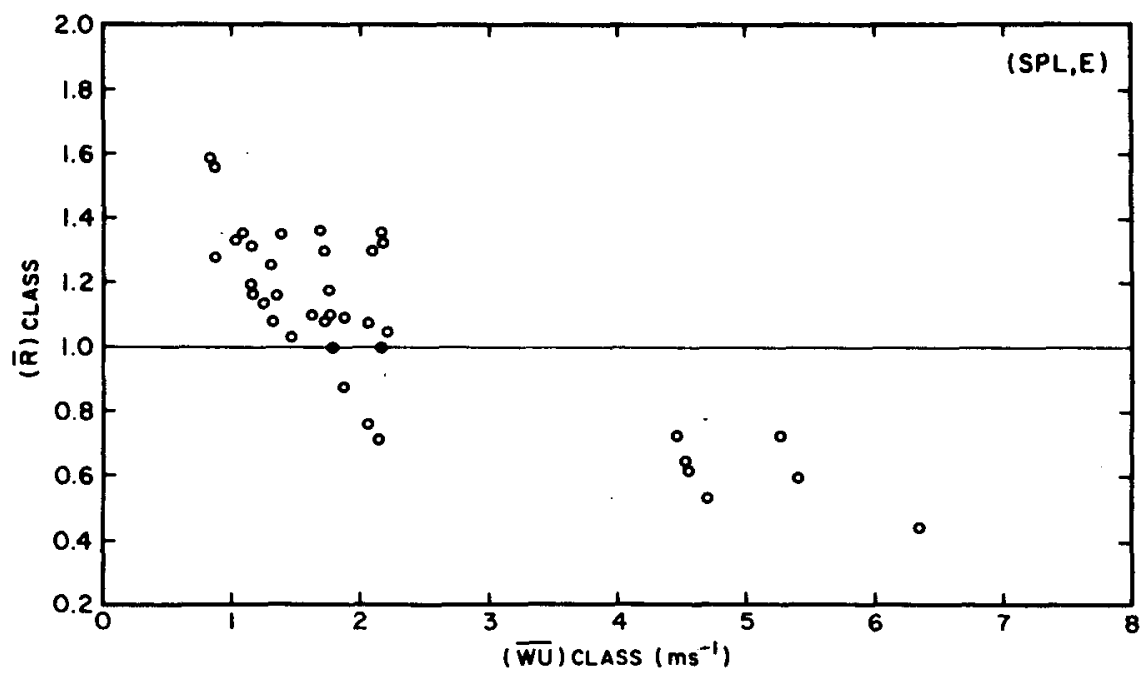

FIG. 15. As in Fig. 14, except for the (SPL, E) profiles. 


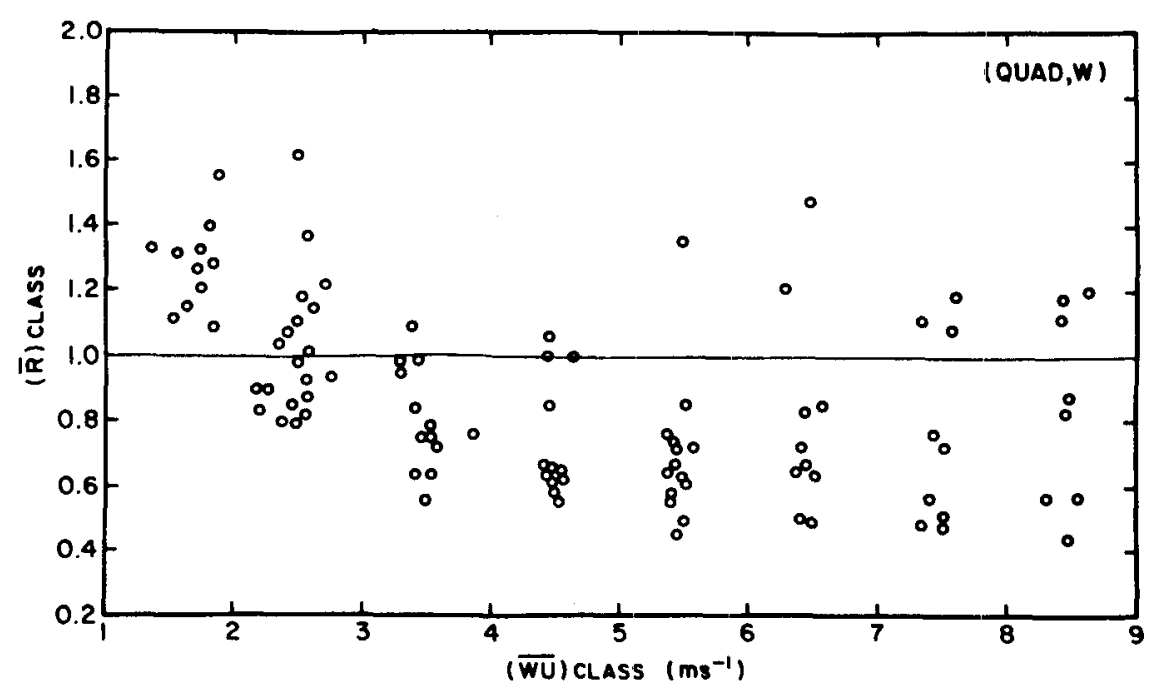

FIG. 16. As in Fig. 14, except for the (QUAD, W) profiles.

obtain useful winds. The stationary sonde experiment has illustrated the impact of smoothing algorithms and base-station motion, and the accuracy and utility of the wind uncertainties.

It was shown that aircraft turns greatly affect the accuracy of real-time (on-board) wind estimates. In the geometric mean, wind error during turns was $50 \%$ higher than during straight and level flight (legs). About half of this increase was due to the shadowing of the Omega antenna during turns, with the remainder due to violation of the assumption of constant aircraft acceleration.

Wind estimates during aircraft turns obtained from cubic-spline phase smoothing were $40 \%$ more accurate than those from low-pass filtering or quadratic smoothing. During the legs, wind errors with the spline were $20-25 \%$ lower than those with the quadratic and filter. Results of the synthetic sounding experiments indicated that quadratic smoothing had the finest resolution of the three methods, although phase error variances of less than $1.5 \mathrm{cec}^{2}$, as well as an absence of turns, are necessary to take advantage of this higher resolution. The noise levels in most soundings, even after editing, produce phase error variances higher than $1.5 \mathrm{cec}^{2}$. We therefore recommend the cubic spline windfinder for nearly all ODW postprocessing.

An estimate of the effect of postprocessing was made by comparing wind error with the quadratic using raw Omega against error with the spline using edited Omega. The postprocessed wind errors were $35 \%$ lower than real-time errors in the legs and 55\% lower in the turns. Thus, highly significant reductions in wind error can be expected through postprocessing.

Results of this study indicated that wind uncertain- ties had been scaled accurately (the factor $K$ in Eq. 12). Sonde-mean uncertainties were useful in estimating the mean wind error in a sounding, but instantaneous uncertainty estimates bore little relation to actual errors and probably should be replaced by the sonde-means in future postprocessed data sets.

Finally, although very low uncertainties were observed in this study $\left(<1 \mathrm{~m} \mathrm{~s}^{-1}\right)$, actual wind errors did not drop as low as the uncertainties. This suggests that sources of error external to the uncertainty calculation may produce noticeable effects when other errors become small; alternatively, the error matrix of phase-rates may not be proportional to the error matrix of the phases alone.

Although much has been learned about Omega wind accuracy and the three phase-smoothing algorithms, many questions still remain. The true effect of turns on cubic-spline winds is hard to gauge with only one flight track, since the classification of "turn" and "leg" points may be meaningless in light of the spline's continuity restrictions. One way to address this question would be to add "aircraft" motion to existing synthetic soundings. With data from stationary and synthetic sondes, progress can be made in the assessment of spline wind accuracy near the edges of soundings. Perhaps the most intriguing questions raised by this research, however, are those concerning the high error ratios for low wind uncertainties, and the possibility of a variable phase-rate to phase error scaling factor. Efforts are under way to address some of these questions.

Acknowledgments. The authors wish to thank the people at the NOAA Office of Aircraft Operations, particularly Jim DuGranrut, for their interest, skill 
and cooperation in the design and execution of the field experiments. Thanks are also due Joe Griffin for his time in the computation of the cubic-spline winds, and to Robert Burpee, Richard Passarelli and Frederick Sanders for their encouragement and valuable suggestions. Finally, we wish to thank Antti Lange, who helped us increase our understanding of the long-path propagation problem.

\section{REFERENCES}

Acheson, D. T., 1974: Omega windfinding and GATE. Bull. Amer. Meteor. Soc., 55, 385-398.

Franklin, J. L., 1983: Omega dropwindsonde processing. NOAA Tech. Memo., ERL-AOML-54, 34 pp.
Govind, P. K., 1975: Omega windfinding systems. J. Appl. Meteor., 14, 1503-15!1.

Julian, P. R., 1982: The aircraft dropwindsonde system in the global weather experiment. Bull. Amer. Meteor. Soc., 73, 619627.

Olson, M. L., R. M. Passi and A. Schumann, 1978: Omega winderror estimation. NCAR/TN-131, $106 \mathrm{pp}$.

Passi, R. M., 1973: Errors in wind measurements derived from Omega signals. NCAR-TR/STR-88, $39 \mathrm{pp}$.

- 1974: Wind determination using Omega signals. J. Appl. Meteor., 13, 934-939.

- 1977 : Smoothing improvement factor in Omega errors. $J$. Appl. Meteor., 16, 735-739.

Shapiro, L. J., 1984: Sampling errors in statistical models of tropical cyclone motion: A comparison of predictor screening and EOF techniques. Mon. Wea. Rev., 112, 1378-1388.

Watt, A. D., 1967: VLF Radio Engineering. Pergamon Press, 703 pp. 